\title{
An Operator Formulation of Orbifold Conformal Field Theory
}

\section{R. Zucchini}

Max-Planck Insitut für Physik und Astrophysik, Werner Heisenberg Institut für Physik, P.O. Box 4012 12, D-8000 Munchen, Federal Republic of Germany

\begin{abstract}
In many applications of conformal field theory one encounters twisted conformal fields, i.e. fields which have branch cut singularities on the relevant Riemann surfaces. We present a geometrical framework describing twisted conformal fields on Riemann surfaces of arbitrary genus which is alternative to the standard method of coverings. We further illustrate the theory of twisted Grassmannians and its relation with the representation theory of the twisted oscillator algebras. As an application of the above, we expound an operator formalism for orbifold strings.
\end{abstract}

\section{Introduction}

In the past few years, there has been great progress toward the formulation of phenomenologically viable string models. All these attempts involve the compactification of the extra dimensions of the heterotic string [1]. The resulting string models are described by superconformally invariant non-linear sigma models [2]. In realistic compactification schemes the conformal field theories involved are interacting and very difficult to deal with [3]. More manageable schemes are based on orbifold backgrounds and the corresponding conformal field theories are exactly solvable [4]. At present, orbifold compactification seems to be the best compromise between computability and phenomenological viability.

It is well-known that the space of classical solutions of string field theory consists of two-dimensional conformally invariant field theories. Therefore, it is important to understand the space of conformal field theories and to develop systematic methods for its study. Within this framework, it is necessary also to understand how to account for quantum effects and carry out non-perturbative analyses. Since string loop effects are described by conformal field theories on higher genus Riemann surfaces, a Grassmannian treatment may provide a unified framework endowed with a topological structure [5].

In this paper we formulate an operator formalism for orbifold strings, on the same line as Alvarez-Gaumé et al. [6]. The possibility of such a generalization of the standard operator formalism was hinted originally by these authors but, to 
the best of our knowledge, never fully developed. This is not a straightforward matter as it may look at first glance, as the nature of our operator formalism forces us to consider sections of the relevant bundles having power branch cuts at prescribed points [7], instead of resorting to the standard method based on the use of suitable coverings [8]. An operator formalism for $\mathbf{Z}_{2}$ orbifold models, based on the method of coverings, has been developed in ref. [9].

Since the essential features of the operator approach have been exhaustively explained in refs. [6], we shall limit ourselves to outline the necessary modifications. The reader is referred to those papers for a clear and selfcontained exposition of the theoretic ideas at the basis of our work. The plan of this paper is as follows. In Sect. (2) we review twisted geometry. In due course, we shall define twisted sections of a line bundle and develop the twisted Serre and Riemann-Roch theory. In Sect. (3) we shall introduce the notion of twisted Grassmannian and define the counterpart of the Krichever map in this context and the state corresponding to a Riemann surface with any number of punctures. In Sect. (4) we shall outline the operator formalism for orbifold strings. In Sect. (5) we discuss briefly possible extensions of our analysis. Finally, in the appendix we collect some useful formulae, which are not directly connected to the main line of development of the paper. Our treatment applies both to the quantum and the instanton part of orbifold amplitudes. We have not attempted a fully supersymmetric formulation, as our understanding of twisted supergeometry is not sufficiently deep yet.

Although some of the conclusions of our work may be expected on the basis of the analogy to the standard operator formalism, there are however original results. We show that all the main theorems of standard algebraic geometry can be suitably generalized to a twisted context and have useful applications to twisted conformal field theory on higher genus Riemann surfaces. We also find that the state $|\Sigma\rangle$ representing an orbifold conformal field theory on a Riemann surface $\Sigma$ is a superposition of states each of which corresponds to an element of a family of parallel hyperplanes of a certain Hilbert space, generalizing the ordinary Grassmannian treatment.

In what follows, we concentrate on the matter sector as this requires a special analysis. In one complex space-time dimension the basic variables are one complex bosonic field $x$ and two complex fermionic fields $\psi$ and $\tilde{\psi}$ defined on a Riemann surface $\Sigma$. The boson field $x$ represents the quantum fluctuation around any instanton background. The dynamics is described by the action

$$
S[x, \psi, \tilde{\psi}]=S_{b}[x]+S_{f}[\psi, \tilde{\psi}],
$$

where

$$
\begin{gathered}
S_{b}[x]=\frac{-i}{8 \pi} \int_{\Sigma}[x \partial \bar{\partial} \bar{x}+\bar{x} \partial \bar{\partial} x], \\
S_{f}[\psi, \tilde{\psi}]=\frac{i}{4 \pi} \int_{\Sigma}[\psi \bar{\psi} \bar{\psi}+\bar{\psi} \bar{\partial} \psi+\tilde{\psi} \partial \tilde{\bar{\psi}}+\tilde{\bar{\psi}} \partial \tilde{\psi}],
\end{gathered}
$$

and $\bar{x}=x^{*}, \bar{\psi}=\tilde{\psi}^{*}, \tilde{\bar{\psi}}=\psi^{*}$. In orbifold models all the above fields must be $t w i s t e d$, i.e. multivalued, on $\Sigma$. As is well-known, field twisting in a path integral formulation 
is equivalent to the insertion in correlation functions of certain vertex operators, called $t$ wist or spin-twist fields, having non-local operator product expansions with the basic fields [7]. In explicit calculations one must use a given determination or sheet of the fields. All physical quantities cannot depend on the specific sheet used i.e. must be invariant under sheet redefinitions. This is in a sense a sort of discrete gauge symmetry. In particular, the global $U(1)$ symmetry of the action ensures that the dynamics is sheet independent. One of the aims of our work is to give a precise geometric definition of these simple physical ideas by means of a manifestly sheet invariant formulation.

\section{Twisted Algebraic Geometry}

As already noticed, the orbifold operator formalism naturally involves twisted conformal fields, i.e. fields which are multivalued. Therefore, a geometrical setting suitable to deal with multivalued fields on Riemann surfaces is necessary [10]. This is outlined below. The proof of the main results is technical and it is sketched in App. A. In what follows, we assume that the reader is familiar with the basics of sheaf theory and complex algebraic geometry [11].

Following the analysis of Alvarez-Gaumé et al. [6], we consider a Riemann surface $\Sigma$ of genus $g$ with $h$ punctures $p_{1}, p_{2}, \ldots, p_{h}$. Occasionally, we shall write $\Sigma_{g, h}$ to emphasize the topological type of the surface $\Sigma$.

We further consider a finite subgroup $\Gamma$ of the circle group $\mathbf{T}$, called the twist group. Any element $\gamma$ of $\Gamma$ can be represented as $\gamma=\exp (-2 \pi i v(\gamma))$, where $v(\gamma)$ is a rational number such that $-1 / 2 \leqq v(\gamma)<1 / 2$, depending on $\gamma \cdot v(\gamma)$ is called the twist of $\gamma$.

A twist structure on $\Sigma$ is an assignment of an element $\gamma(l)$ of $\Gamma$ to any homotopy loop $l$ of $\pi_{1}(\Sigma)$. Twist structures describe the multivaluedness of smooth maps of $\Sigma$ into the one-dimensional orbifold $S^{1} / \Gamma$ when viewed as maps of $\Sigma$ into $S^{1}$. A simple analysis shows that twist structures are homomorphisms of the first homology group $H_{1}(\Sigma, \mathbf{Z})$ into $\Gamma$. Therefore, any twist structure $\gamma$ is completely determined by specifying the twists $v(l, \gamma)$ corresponding to each homology loop $l$ of a set of generators of $H_{1}(\Sigma, Z)$. We shall denote by $v_{k}(\gamma)$ the twist associated to the homology loop which only winds once around $p_{k}$ anticlockwise with respect to a chosen orientation of $\Sigma$. Since the homology loop which only winds once around each puncture is trivial. the total twist

$$
n(\gamma)=\sum_{k=1}^{h} v_{k}(\gamma)
$$

is an integer number. Often, we shall write $\Sigma(\gamma)$ to evidentiate that $\Sigma$ is endowed with the twist structure $\gamma$.

In what follows, we shall first consider special twist structures, i.e. twist structures $\gamma$ whose only possibly non-vanishing twists are the $v_{k}(\gamma)$ 's. Later, we shall explain how to extend our analysis to arbitrary twist structures.

We shall now tackle the problem of defining twisted fields on $\Sigma$. We assume that the multivaluedness of the fields is described by a special twist structure $\gamma$, i.e. that the fields have a power branch cut of the form $z_{k}^{\mp v_{k}(\gamma)}$ at the $k^{\text {th }}$ puncture, 
where $z_{k}$ is a holomorphic coordinate defined in the neighbourhood of the $k^{\text {th }}$ puncture and vanishing at the puncture. We further assume that these fields are sections of some type of a holomorphic line bundle $\xi$ on $\Sigma_{c}$, where $\Sigma_{c}$ is the compactification of $\Sigma$, i.e. the Riemann surface obtained by filling the punctures of $\Sigma$. The information contained in the above assumptions is only local, that is it states the properties of local germs of twisted fields. We are interested conversely in globally defined twisted fields. Therefore, we need a systematic method to study the ways germs of fields can be assembled to yield globally defined fields. As is well-known, this is provided by sheaf cohomology theory. The sheaves we shall define below are all supported on $\Sigma_{c}$. In fact, the punctures are not really deleted points but serve only the purpose of defining the boundary conditions obeyed by the twisted fields.

From the above, it follows that the relevant sheaves describing twisted fields are

$$
\begin{gathered}
\mathcal{O}^{ \pm}(\gamma)_{p}=\mathbf{C}\{z\}, \quad p \neq p_{k}, \\
\mathcal{O}^{ \pm}(\gamma)_{p_{k}}=\mathbf{C}\left\{z_{k}\right\} \otimes z_{k}^{\mp} v_{k}(\gamma),
\end{gathered}
$$

$\mathbf{C}\{z\}$ being the ring of holomorphic functions of the complex variable $z$. The sheaf of germs of twisted holomorphic functions $\mathcal{O}^{ \pm}(\gamma)$ can be topologized in standard fashion by assigning a system of neighbourhoods of each germ $\phi$ at each point $p$ of $\Sigma_{c}$. Given any function $f$, defined in a neighbourhood of $p$, with the regularity properties specified by $(2.2 \mathrm{a}-\mathrm{b})$ and such that $f_{p}=\phi$, define a neighbourhood of $\phi$ as the set of all germs $f_{q}$ with $q$ in the domain of $f$. Clearly, this neighbourhood depends on the choice of $f$. A system of neighbourhoods is then obtained by varying $f$ in compliance with the above restrictions.

The \pm -twisted holomorphic fields are just the continuous sections of the sheaf

$$
\mathcal{O}^{ \pm}(\xi, \gamma)=\mathcal{O}(\xi) \otimes \mathcal{O}^{ \pm}(\gamma)
$$

where $\mathcal{O}(\xi)$ is the ordinary sheaf of germs of holomorphic sections of the line bundle $\xi$. They form a space given by

$$
\Gamma\left(\Sigma_{c}, \mathcal{O}^{ \pm}(\xi, \gamma)\right)=H^{0}\left(\Sigma_{c}, \mathcal{O}^{ \pm}(\xi, \gamma)\right)
$$

The Serre duality and Riemann-Roch theorems generalize to the present setting. The twisted Serre theorem states

$$
H^{1}\left(\Sigma_{c}, \mathcal{O}^{ \pm}(\xi, \gamma)\right) \equiv H^{0}\left(\Sigma_{c}, \mathcal{O}^{\mp}\left(\kappa \otimes \xi^{-1}, \gamma\right)\right)^{V},
$$

where $\kappa$ is the canonical line bundle and the superscript $V$ denotes duality of vector spaces. The twisted Riemann-Roch theorem reads

$$
\operatorname{dim} H^{0}\left(\Sigma_{c}, \mathcal{O}^{ \pm}(\xi, \gamma)\right)-\operatorname{dim} H^{1}\left(\Sigma_{c}, \mathcal{O}^{ \pm}(\xi, \gamma)\right)= \pm n(\gamma)+c_{1}(\xi)-g+1,
$$

where $c_{1}(\xi)$ is the first Chern class of $\xi$. Note that the twisted Riemann-Roch index differs from the usual index by an amount determined uniquely by the twist structure $\gamma$ and independent from both the holomorphic line bundle $\xi$ and the genus of $\Sigma$. Mimicking the standard Riemann-Roch theory, it can be shown that in general

$$
\operatorname{dim} \Gamma\left(\Sigma_{c}, \mathcal{O}^{ \pm}(\xi, \gamma)\right)=\max \left(0, \pm n(\gamma)+c_{1}(\xi)-g+1\right)
$$


The result is strictly true only if either $\pm n(\gamma)+c_{1}(\xi)<0$ or $2 g-2< \pm n(\gamma)+c_{1}(\xi)$, but it holds also when $0 \leqq \pm n(\gamma)+c_{1}(\xi) \leqq 2 g-2$ for a generic location of the punctures and a generic line bundle $\xi$. In the following, we shall always assume the generic situation, although we should always be aware of possible exceptions.

Meromorphic twisted fields are treated similarly. In this case the relevant sheaf is

$$
\mathscr{M}^{ \pm}(\xi, \gamma)=\mathscr{M}(\xi) \otimes \mathcal{O}^{ \pm}(\gamma)
$$

where $\mathscr{M}(\xi)$ is the ordinary sheaf of germs of meromorphic sections of $\xi$. The \pm -twisted meromorphic fields are just the continuous sections of the sheaf $\mathscr{M}^{ \pm}(\xi, \gamma)$. They form a space given by

$$
\Gamma\left(\Sigma_{c}, \mathscr{M}^{ \pm}(\xi, \gamma)\right)=H^{0}\left(\Sigma_{c}, \mathscr{M}^{ \pm}(\xi, \gamma)\right) .
$$

As in the standard case, twisted meromorphic fields cannot have poles of arbitrary order at the punctures. Indeed, the orders of these poles are restricted by the twisted Weierstrass gap theorem. If $q_{1}, q_{2}, \ldots, q_{r}$ are $r$ points of $\Sigma$ (part or all of which may coincide with the punctures) and $n_{1}, n_{2}, \ldots, n_{r}$ are $r$ integers, the dimension of the space of sections of $\mathscr{M}^{ \pm}(\xi, \gamma)$ having poles only at the $q_{k}$ 's of order at most $n_{k}$ is given by

$$
\operatorname{dim} \Gamma\left(\Sigma_{c}, \mathcal{O}^{ \pm}(\xi, \gamma) \otimes \mathcal{O}\left(\sum_{k=1}^{r} n_{k} q_{k}\right)\right)=\max \left(0, \pm n(\gamma)+\sum_{k=1}^{r} n_{k}+c_{1}(\xi)-g+1\right)
$$

in the generic situation (cfr. Eq. (2.7)). As in the ordinary operator formalism, the knowledge of the gap structure of the meromorphic sections of the relevant bundles plays an important role.

It is easy to see that the sheaves $\mathscr{M}^{ \pm}(\xi, \gamma)$ and $\mathscr{M}^{\mp}\left(\xi, \gamma^{-1}\right)$ are isomorphic, unlike their holomorphic counterparts. However, when applying the identity (2.9) we should be aware that the definition of order of a pole may differ by a unit in the two sheaves.

As an application of the above results, we consider the important cases where either $\xi$ is the trivial line bundle 1 or one of the $2^{2 g}$ spin bundles $\lambda$. From (2.7), we see that the number of linearly independent \pm -twisted holomorphic functions is given by $\max ( \pm n(\gamma)-g+1,0)$ in the generic situation. Note that +- and - -twisted holomorphic functions cannot exist simultaneously if $n(\gamma) \neq 0$. We have verified this result by explicitly constructing a basis of \pm -twisted holomorphic functions for $|n(\gamma)| \geqq g$ and $\operatorname{sign} n(\gamma)= \pm 1$ (cfr. $(A .10 \mathrm{c})$ ). The above result fails to hold for $n(\gamma)=0$ and $g>0$, as it does in the standard case. Similarly, we see that the number of linearly independent \pm -twisted holomorphic spinors is $\max ( \pm n(\gamma), 0)$. Thus, the number of twisted holomorphic spinors is determined entirely by the total twist and, as in the bosonic case +- and - -twisted holomorphic spinors cannot exist at the same time. We have verified also this result by explicitly constructing a basis of \pm -holomorphic spinors for $|n(\gamma)|>0$ and $\operatorname{sign} n(\gamma)= \pm 1$ (cfr. $(A .10 \mathrm{~d})$ ). The above result does not hold for an odd spin structure for $n(\gamma)=0$ and $g>0$, as in the standard case.

Next, we shall introduce the notion of twisted harmonic function and 
differential. The justification of the definitions given below will be provided later. The $\pm-t$ wisted harmonic functions are defined as the elements of the space of sections

$$
H^{ \pm}(\Sigma, \gamma)=\Gamma\left(\Sigma_{c}, \mathcal{O}^{ \pm}(1, \gamma) \otimes \mathcal{O}\left(\sum_{l=1}^{h}\left[\mp v_{l}(\gamma)\right] p_{l}\right) \oplus \overline{\mathcal{O}^{\mp}(1, \gamma) \otimes \mathcal{O}\left(\sum_{l=1}^{h}\left[ \pm v_{l}(\gamma)\right] p_{l}\right)}\right)
$$

where the bar denotes the ordinary complex conjugation of sections. The dimension of $H^{ \pm}(\Sigma, \gamma)$ is 0 if $\gamma \neq 1$ and 1 otherwise. In the following we shall consider mainly \pm -twisted functions which are harmonic off the punctures. These are the elements of the spaces $H_{n m}^{ \pm}(\Sigma, \gamma)$ of continuous sections of the sheaves obtained by tensoring the sheaf appearing in the right-hand side of Eq. (2.11) by the sheaves $\mathcal{O}\left(\sum_{l=1}^{h} m_{l} p_{l}\right) \otimes \mathcal{O}\left(\sum_{l=1}^{h} n_{l} p_{l}\right)$, where the $m_{l}$ 's and $n_{l}$ 's are arbitrary non-negative integers. The dimension of $H_{n m}^{ \pm}(\Sigma, \gamma)$ is given by $\operatorname{dim} H^{ \pm}(\Sigma, \gamma)+\sum_{l=1}^{h} m_{l}+\sum_{l=1}^{h} n_{l}$, that is there are no gaps. This can be proven by constructing a generating kernel whose derivatives with respect to the second argument provide a basis of the above space (the kernel happens to be the correlation function of the fields $x$ and $\bar{x}$ which has been computed in ref. [12]). The \pm -twisted harmonic forms are similarly defined as the elements of the space

$$
K^{ \pm}(\Sigma, \gamma)=\Gamma\left(\Sigma_{c}, \mathcal{O}^{ \pm}(\kappa, \gamma) \otimes \mathcal{O}\left(-\sum_{l=1}^{h}\left[ \pm v_{l}(\gamma)\right] p_{l}\right) \oplus \mathcal{O}^{\mp}(\kappa, \gamma) \otimes \mathcal{O}\left(-\sum_{l=1}^{h}\left[\mp v_{l}(\gamma)\right] p_{l}\right)\right)
$$

From the twisted gap theorem it follows that the dimension of $K^{ \pm}(\Sigma, \gamma)$ is $2 g-2+h(\gamma)$, where $h(\gamma)$ is the number of punctures of $\Sigma$ such that $v_{l}(\gamma) \neq 0$, if $\gamma \neq 1$, and $2 g$ otherwise [12]. It is also convenient to introduce the \pm -twisted forms which are harmonic off the punctures. As for the functions, these are the elements of the spaces $K_{n m}^{ \pm}(\Sigma, \gamma)$ of continuous sections of the sheaves obtained by tensoring the sheaf in the right-hand side of Eq. (2.12) by the sheaves $\mathcal{O}\left(\sum_{l=1}^{h} m_{l} p_{l}\right) \otimes \mathcal{O}\left(\sum_{l=1}^{h} n_{l} p_{l}\right)$ for arbitrary non-negative integers $m_{l}$ and $n_{l}$. The dimension of $K_{n m}^{ \pm}(\Sigma, \gamma)$ is given by $\operatorname{dim} K^{ \pm}(\Sigma, \gamma)+\sum_{l=1}^{h} m_{l}+\sum_{l=1}^{h} n_{l}$.

Note that $H_{n m}^{ \pm}(\Sigma, \gamma)$ is always contained in $\Gamma\left(\Sigma_{c}, \mathscr{M}^{ \pm}(1, \gamma) \oplus \overline{\left.\mathscr{M}^{\mp}(1, \gamma)\right)}\right.$, but not in $\Gamma\left(\Sigma_{c}, \mathscr{M}^{ \pm}(1, \gamma)\right) \oplus \Gamma\left(\Sigma_{c}, \overline{\mathscr{M}}^{\mp}(1, \gamma)\right)$ in general, because of the restrictions imposed by the gap theorem on the latter space. However, it is always possible to express a twisted harmonic function with poles as a sum of an intrinsically multi-valued meromorphic and antimeromorphic function. We have to explain clearly the meaning of intrinsic multivaluedness. In fact, twisted functions are multivalued in any case around the punctures of $\Sigma$. A function is intrinsically multivalued when its variation around any loop of zero $(\bmod Z)$ total twist is non-zero. These loops form a complex vector space which may be considered a twisted generalization 
of the ordinary first homology. Its dimension is $2 g-2+h(\gamma)$ if $\gamma \neq 1$ and $2 g$ otherwise $[7,12]$. The space of zero total twist loops is naturally identified with the dual $K^{ \pm}(\Sigma, \gamma)^{V}$ of $K^{ \pm}(\Sigma, \gamma)$. As usual, the duality is defined by

$$
\langle\lambda, \omega\rangle=\oint_{\lambda} \omega,
$$

where $\lambda \in K^{ \pm}(\Sigma, \gamma)^{V}$ and $\omega \in K^{ \pm}(\Sigma, \gamma)$. These results partly justify the above definitions and will be useful in studying the operator formalism.

The natural question arises about whether the (anti)holomorphic sections of the bundles 1 and $\lambda$ correspond to the solutions of the classical equations of motion associated with the action (1.1a). In the following discussion we shall assume for simplicity that all the twists $v_{k}(\gamma)$ are different from $-1 / 2$. The modifications necessary in the case where some twists take this exceptional value are left to reader.

Let us introduce the sheaves

$$
\mathscr{E}^{ \pm}(\xi, \gamma)=\mathscr{E}(\xi) \otimes \mathcal{O}^{ \pm}(\gamma)
$$

where $\mathscr{E}(\xi)$ is the sheaf of germs of smooth sections of the line bundle $\xi$. The continuous sections of $\mathscr{E}^{ \pm}(\xi, \gamma)$ are just the \pm -twisted smooth fields. A simple analysis based on the power counting of the possible integration singularities shows that the domain of finiteness for the bosonic action $S_{b}[x]$ (cfr. (1.1b)) is

$$
\mathbf{B}(\Sigma, \gamma)=\Gamma\left(\Sigma_{c}, \mathscr{E}^{+}(1, \gamma) \oplus \overline{\left.\mathscr{E}^{-}(1, \gamma)\right)} .\right.
$$

Similarly, the domain of finiteness for the fermionic action $S_{f}[\psi, \tilde{\psi}]$ (cfr. (1.1c)) is

$$
\mathbf{F}(\Sigma, \gamma)=\Gamma\left(\Sigma_{c}, \mathscr{E}^{+}(\lambda, \gamma) \oplus \overline{\left.\mathscr{E}^{-}(\bar{\lambda}, \gamma)\right)} \otimes \mathbf{G} \times \Gamma\left(\Sigma_{c}, \mathscr{E}^{+}(\bar{\lambda}, \gamma) \oplus \overline{\left.\mathscr{E}^{-}(\lambda, \gamma)\right)} \otimes \mathbf{G},\right.\right.
$$

where $\bar{\lambda}$ denotes the complex conjugate of the line bundle $\lambda$ and $\mathbf{G}$ is the fermionic Grassmann algebra. The two factors of the right-hand side of $(2.16 \mathrm{~b})$ are the domains of the two independent fermionic fields. In addition to finiteness, we must require that the bosonic Laplacian operator $\partial \bar{\partial}$ and the fermionic Cauchy-Riemann operator $\partial$ have a well-defined adjoint. In the bosonic case, this entails the further restriction

$$
\sum_{k=1}^{h} v_{k}(\gamma) \operatorname{Im}\left(x_{k}^{+} x_{k}^{-}\left(p_{k}\right)\right)=0,
$$

where the $x_{k}^{ \pm}$'s are smooth functions such that

$$
x(p)=x_{k}^{+}(p) z_{k}(p)^{-v_{k}(\gamma)}+\bar{x}_{k}^{-}(p) \bar{z}_{k}(p)^{v_{k}(\gamma)}
$$

near $p_{k}, z_{k}(p)$ being a local holomorphic coordinate vanishing at $p_{k}$. There are no additional restrictions in the fermionic case. In orbifold conformal field theory, condition (2.16a) is satisfied by imposing the natural condition $x_{k}^{ \pm}\left(p_{k}\right)=0$ for $\pm v_{k}(\gamma)>0$ [7]. This restriction eliminates all twisted holomorphic functions, which therefore cannot be considered solutions of the classical equations of motion. The only allowed bosonic zero modes are the twisted harmonic functions which exist only in the trivial case $\gamma=1$ (see the discussion following Eq. (2.11)). Conversely, the twisted holomorphic spinors are really fermionic zero modes and their existence 
corresponds to the presence of a twist background charge which must be accounted for in a consistent path integral formulation. This point should be emphasized.

We now shall comment on the extension of the previous results to arbitrary twist structures. We note that twists around the non-trivial homology loops $\Sigma_{c}$ can be enforced by tensoring the relevant line bundles with an appropriate flat line bundle [11]. Thus, a general twists structure can be viewed as a pair $(\phi, \gamma)$ formed by a flat line bundle $\phi$ and a special twist structure $\gamma$. All the above theorems generalize straightforwardly by replacing a general line bundle $\xi$ by $\xi \otimes \phi$. Note in particular that the twisted Riemann-Roch index is not altered by this substitution as $c_{1}(\phi)=0$.

We conclude this section by illustrating an application of the above theorems. Correlators with twist field insertions have been computed by several authors $[7-8,12]$. In particular, in the fermionic case the bosonization formulae can be used [13]. The twisted gap theorem allows an economical derivation and provides an original geometric interpretation of those formulae. Assume for definiteness that $n(\gamma)>0$. Then, according to the above discussion, there are $n(\gamma)$ fermionic zero modes. Thus, the only non-zero twisted fermion correlators are of the form

$$
\left\langle\psi\left(q_{1}\right) \cdots \psi\left(q_{N+n(\gamma)}\right) \bar{\psi}\left(r_{1}\right) \cdots \bar{\psi}\left(r_{N}\right)\right\rangle .
$$

The operator product expansions are

$$
\begin{aligned}
\psi\left(q_{i}\right) \psi\left(q_{j}\right) & \sim z\left(q_{i}\right)-z\left(q_{j}\right), \\
\bar{\psi}\left(r_{i}\right) \bar{\psi}\left(r_{j}\right) & \sim z\left(r_{i}\right)-z\left(r_{j}\right), \\
\psi\left(q_{i}\right) \bar{\psi}\left(r_{j}\right) & \sim\left(z\left(q_{i}\right)-z\left(r_{j}\right)\right)^{-1} .
\end{aligned}
$$

The monodromy at the punctures is

$$
\begin{aligned}
& \psi\left(q_{i}\right) \sim\left(z\left(q_{i}\right)-z\left(p_{k}\right)\right)^{-v_{k}(\gamma)}, \\
& \bar{\psi}\left(r_{j}\right) \sim\left(z\left(r_{j}\right)-z\left(p_{k}\right)\right)^{v_{k}(\gamma)},
\end{aligned}
$$

while the monodromy around the homology cycles of $\Sigma_{c}$ is trivial. The bosonization formula for the correlator with twist field insertions corresponding to $(2.17 \mathrm{a})$ is a trivial solution of the above operator product expansions and monodromy conditions. We have to prove that it is the only solution up to a multiplicative factor. If we allow only $q_{i}$ to vary and keep all the other insertion points $q_{j}, j \neq i$ and $r_{k}$ fixed, then the correlator (2.17a) is a section of the sheaf $\mathcal{O}^{+}(\lambda, \gamma) \otimes$ $\mathcal{O}\left(-\sum_{j=1, j \neq i}^{N+n(\gamma)} q_{j}+\sum_{k=1}^{N} r_{k}\right)$. By the twisted gap theorem there is only one continuous section of such a sheaf. A similar reasoning applies also if we consider the correlator (2.17a) as a function of $r_{k}$ only. Thus the bosonization formula is the only possible solution of the operator product expansions and monodromy conditions $(2.17 \mathrm{~b}-\mathrm{f})$.

\section{Twisted USp- and U-Grassmannians}

In the standard operator formalism an important role is played by Grassmannians, as they provide a unified framework for the study of conformal field theories on 
higher genus Riemann surfaces $[5,6]$. One may expect that in the orbifold operator formalism a similar role is played by twisted Grassmannians. This is indeed the case. We shall now outline the theory of twisted USp- and $U$-Grassmannians, as these are relevant for the study of world-sheet bosons and fermions for string propagating on a orbifold background.

We consider a set of rational numbers $\tau_{j}, j=1, \ldots, h$ such that $-1 / 2 \leqq \tau_{j}<1 / 2$. Eventually these will be identified with the twists of a given twist structure. However, in the following analysis this assumption is not necessary.

We examine first orbifold bosons. We define $L(\tau)$ as the complex vector space of all formal power series

$$
\begin{gathered}
\xi^{j}\left(z_{j}, \bar{z}_{j}\right)=\sum_{r=-\infty}^{\infty}\left[\frac{1}{r-\tau_{j}} \xi_{r-\tau_{j}}^{j} z_{j}^{r-\tau_{j}}+\frac{1}{r+\tau_{j}} \tilde{\xi}_{r+\tau_{j}}^{j} \bar{z}_{j}^{r+\tau_{j}}\right], \\
\sum_{r=-\infty}^{\infty}\left[\left|\frac{1}{r-\tau_{j}}\right|\left|\xi_{r-\tau_{j}}^{j}\right|^{2}+\left.\left|\frac{1}{r+\tau_{j}}\right| \tilde{\xi}_{r+\tau_{j}}^{j}\right|^{2}\right]<\infty .
\end{gathered}
$$

Here and in the following we assume without statement that any quantity with an index $m \pm \tau_{j}$ either vanishes or is absent whenever this index vanishes. This convention will save us a lot of writing and will also simplify our formulae.

The space $L(\tau)$ can be given the structure of complex Hilbert space by means of the norm defined by the expression (3.1b). This structure is then used to provide $L(\tau)$ with a suitable topology.

The elements of $L(\tau)$ represent the oscillatory part of the classical configurations of strings belonging to $h$ twisted sectors with twists specified by the $\tau_{j}$ 's. Of course, the acutal configurations which appear in string perturbation theory correspond to certain proper subspaces of $L(\tau)$ depending on the Riemann surfaces involved. This will be explained in greater detail later.

It is possible to endow $L(\tau)$ with a non-singular antihermitian sesquilinear form, which renders it a unisymplectic space. Such form is essentially the integral expression of the charge associated with the global $U(1)$ symmetry of the bosonic action $S_{b}[x]$ (cfr. Eq. (1.1b)),

$$
\begin{aligned}
\Phi_{b}(\xi, \bar{\eta}) & =\sum_{j=1}^{h} \frac{1}{4 \pi i} \oint_{S^{1}}\left[\xi^{j}\left(\partial_{j}-\bar{\partial}_{j}\right) \bar{\eta}^{j}-\bar{\eta}^{j}\left(\partial_{j}-\bar{\partial}_{j}\right) \xi^{j}\right] \\
& =\sum_{j=1}^{h} \sum_{r=-\infty}^{\infty}\left[\frac{1}{r-\tau_{j}} \xi_{r-\tau_{j}}^{j} \overline{\tilde{\eta}_{-r+\tau_{j}}^{j}}+\frac{1}{r+\tau_{j}} \tilde{\xi}_{r+\tau_{j}}^{j} \overline{\eta_{-r-\tau_{j}}^{j}}\right] .
\end{aligned}
$$

We denote by $U S p(L(\tau))$ the group of continuous unisymplectic linear operators on $L(\tau)$.

Next, we introduce a set of operators $\alpha_{m-\tau_{j}}^{j}, \tilde{\alpha}_{m+\tau_{j}}^{j}, \bar{\alpha}_{m+\tau_{j}}^{j}$ and $\tilde{\alpha}_{m-\tau_{j}}^{j}$, obeying the commutation relations

$$
\begin{aligned}
& {\left[\alpha_{m-\tau_{j}}^{j}, \bar{\alpha}_{n+\tau_{k}}^{k}\right]_{-}=-2\left(m-\tau_{j}\right) \delta_{m+n, 0} \delta^{j k},} \\
& {\left[\tilde{\alpha}_{m+\tau_{j}}^{j}, \tilde{\tilde{\alpha}}_{n-\tau_{k}}^{k}\right]_{-}=-2\left(m+\tau_{j}\right) \delta_{m+n, 0} \delta^{j k},}
\end{aligned}
$$


the remaining commutators being zero, and the adjunction relations

$$
\begin{aligned}
& \alpha_{m-\tau_{j}}^{j}=-\bar{\alpha}_{-m+\tau_{j}}^{j}, \\
& \tilde{\alpha}_{m+\tau_{j}}^{j}=-\tilde{\bar{\alpha}}_{-m-\tau_{j}}^{j} .
\end{aligned}
$$

As is well-known, these operators define the Fock representations associated to $h$ twisted sectors with twists $\tau_{j}$. The oscillatory part of the bosonic field operators can be expressed in terms of the $\alpha$ operators:

$$
\begin{aligned}
& x^{j}\left(z_{j}, \bar{z}_{j}\right)=\sum_{r=-\infty}^{\infty}\left[\frac{1}{r-\tau_{j}} \alpha_{r-\tau_{j}}^{j} z_{j}^{r-\tau_{j}}+\frac{1}{r+\tau_{j}} \tilde{\alpha}_{r+\tau_{j}}^{j} \bar{z}_{j}^{r+\tau_{j}}\right], \\
& \bar{x}^{j}\left(z_{j}, \bar{z}_{j}\right)=\sum_{r=-\infty}^{\infty}\left[\frac{1}{r+\tau_{j}} \bar{\alpha}_{r+\tau_{j}^{j}}^{j} z_{j}^{r+\tau_{j}}+\frac{1}{r-\tau_{j}} \tilde{\bar{\alpha}}_{r-\tau_{j}}^{j} \bar{z}_{j}^{r-\tau_{j}}\right] .
\end{aligned}
$$

We can use any element $\xi$ of $L(\tau)$ to smear the field operators

$$
\begin{aligned}
& x(\bar{\xi})=-\Phi_{b}(x, \bar{\xi})=\sum_{j=1}^{h} \sum_{r=-\infty}^{\infty}\left[\frac{1}{r+\tau_{j}} \overline{\tilde{\xi}_{r+\tau_{j}}^{j}} \alpha_{-r-\tau_{j}}^{j}+\frac{1}{r-\tau_{j}} \overline{\xi_{r-\tau_{j}}^{j}} \tilde{\alpha}_{-r+\tau_{j}}^{j}\right], \\
& \bar{x}(\xi)=\Phi_{b}(\xi, \bar{x})=\sum_{j=1}^{h} \sum_{r=-\infty}^{\infty}\left[\frac{1}{r-\tau_{j}} \xi_{r-\tau_{j}}^{j} \bar{\alpha}_{-r+\tau_{j}}^{j}+\frac{1}{r+\tau_{j}} \tilde{\xi}_{r+\tau_{j}}^{j} \tilde{\bar{\alpha}}_{-r-\tau_{j}}^{j}\right] .
\end{aligned}
$$

The operators $x(\bar{\xi})$ and $\bar{x}(\xi)$ obey the algebra

$$
[\bar{x}(\xi), x(\bar{\eta})]_{-}=-2 \Phi_{b}(\xi, \bar{\eta})
$$

for $\xi, \eta \in L(\tau)$, the remaining commutators being zero.

From here, it is apparent that the maximal commuting subalgebras of the algebra (3.6) correspond to the maximal isotropic subspaces of the unisymplectic space $L(\tau)$. We wish to define the manifold of these subspaces in a more precise fashion $[5,14]$. We consider a polarization of $L(\tau)$ as a Hilbert space

$$
L(\tau)=L_{+} \oplus L_{-},
$$

where $L_{ \pm}$are maximal isotropic subspaces of $L(\tau)$. Now, any continuous operator $A$ in $L(\tau)$ can be written as

$$
A=\left(\begin{array}{ll}
A_{++} & A_{+-} \\
A_{-+} & A_{--}
\end{array}\right)
$$

with respect to this direct sum decomposition. We denote by $U S p_{\text {res }}(L(\tau))$ the subgroup of $U S p(L(\tau))$ formed by all the elements $U$ of $U S p(L(\tau))$ such that $U_{+}$and $U_{-+}$are Hilbert-Schmidt operators in $L(\tau)$. The twisted Grassmannian manifold $\operatorname{USp}(L(\tau))$ is the homogeneous space

$$
\mathrm{USp}(L(\tau))=\frac{U S p_{\mathrm{res}}(L(\tau))}{\left[G L\left(L_{+}\right) \times G L\left(L_{-}\right)\right]_{\mu}},
$$

where the two factors in the denominator are identified by means of the isomorphism $\mu: G L\left(L_{+}\right) \mapsto G L\left(L^{-}\right), \mu\left(U_{++}\right)=U_{++}^{-1} \dagger$ and the adjunction operation is with respect to the non-singular bilinear pairing of $L_{+} \times L_{-}$induced by the unisymplectic space $L(\tau)$. 
$\mathrm{USp}(L(\tau))$ can be interpreted as the manifold of all maximal isotropic subspaces $W$ of $L(\tau)$ which do not differ too strongly from $L_{+}$[6]. Thus, $\operatorname{USp} L(\tau)$ does not contain all maximal isotropic subspaces of $L(\tau)$ and in fact depends on $L_{+}$, even though we may replace $L_{+}$by any element of $\operatorname{USp}(L(\tau))$ in (3.8). So, we have to specify the choice of $L_{+}$we shall use in the following. We define $L_{+}$as the subspace of all $\xi \in L(\tau)$ such that $\xi_{m-\tau_{j}}^{j}=0$ for $m>\tau_{j}$ and $\tilde{\xi}_{m+\tau_{j}}^{j}=0$ for $m \geqq-\tau_{j}$ and $L_{-}$as its orthogonal complement. It is easily checked that $L_{ \pm}$are maximal isotropic subspaces of $L(\tau)$. The reason for this definition will become clear shortly.

Since any element of $\operatorname{USp}(L(\tau))$ is a subspace of $L(\tau)$, it is completely determined by specifying one of its bases. This basis can always be chosen in the following standard form. For any $j=1, \ldots, h$, let $I_{j}$ be a set of integers such that $I_{j} \backslash \mathbf{N}$ and $\mathbf{N} \backslash I_{j}$ are finite sets. This means that $I_{j}$ contains only a finite number of negative integers and all positive integers but a finite number. Let further

$$
\begin{aligned}
\eta_{-m-\tau_{j}}^{j}\left(z_{k}, \bar{z}_{k}\right)= & \delta^{j k} z_{k}^{-m-\tau_{k}}+\sum_{r \in I_{k}} R_{m+\tau_{j}, r-\tau_{k}}^{j k} \frac{1}{r-\tau_{k}} z_{k}^{r-\tau_{k}} \\
& +\sum_{s \in-I_{k}} S_{m+\tau_{j}, s+\tau_{k}}^{j k} \frac{1}{S+\tau_{k}} \bar{z}_{k}^{s+\tau_{k}}, \quad m \in \overline{-I_{j}}, \\
\tilde{\eta}_{-m+\tau_{j}}^{j}{ }^{k}\left(z_{k}, \bar{z}_{k}\right)= & \delta^{j k} \bar{z}_{k}^{-m+\tau_{k}}+\sum_{s \in I_{k}} \tilde{S}_{m-\tau_{j}, s-\tau_{k}}^{j k} \frac{1}{s-\tau_{k}} z_{k}^{s-\tau_{k}} \\
& +\sum_{r \in \frac{I_{k}}{R_{m-\tau_{j}, r+\tau_{k}}} \frac{1}{r+\tau_{k}} \bar{z}_{k}^{r+\tau_{k}}, \quad m \in I_{j},}
\end{aligned}
$$

where $\overline{-I_{j}}$ denotes the complementary set of the set formed by the negative of the elements of the set $I_{j}$,

$$
\begin{aligned}
& R_{m+\tau_{\jmath}, n-\tau_{k}}^{j k}=\overline{\widetilde{R}_{n-\tau_{k}, m+\tau_{j}}^{k j}}, \\
& S_{m+\tau_{\jmath}, n+\tau_{k}}^{j k}=\overline{S_{n+\tau_{k}, m+\tau_{\jmath}}^{k j}} \\
& \tilde{S}_{m-\tau_{j}, n-\tau_{k}}^{j k}=\overline{\widetilde{S}_{n-\tau_{k}, m-\tau_{j}}^{k j}},
\end{aligned}
$$

and

$$
\begin{aligned}
& \sum_{r \in-I_{j}, s \in I_{k}}\left|\frac{1}{r+\tau_{j}} \frac{1}{s-\tau_{k}}\right|\left|R_{r+\tau_{j}, s-\tau_{k}}^{j k}\right|^{2}+\sum_{r \in-I_{j}, s \in-I_{k}}\left|\frac{1}{r+\tau_{j}} \frac{1}{s+\tau_{k}}\right|\left|S_{r+\tau_{j}, s+\tau_{k}}^{j k}\right|^{2} \\
& \quad+\sum_{r \in I_{j}, s \in-I_{k}}\left|\frac{1}{r-\tau_{j}} \frac{1}{s+\tau_{k}}\right|\left|\tilde{R}_{r-\tau_{j}, s+\tau_{k}}^{j k}\right|^{2}+\sum_{r \in I_{j}, s \in I_{k}}\left|\frac{1}{r-\tau_{j}} \frac{1}{s-\tau_{k}}\right|\left|\tilde{S}_{r-\tau_{j}, s-\tau_{k}}^{j k}\right|^{2}<\infty .
\end{aligned}
$$

From (3.9a-e), it follows that the $\eta$ 's and $\tilde{\eta}$ 's span a maximal isotropic subspace of $L(\tau)$. The condition (3.9f) ensures further that this subspace belongs to $\mathbf{U S p}(L(\tau))$. We remark that any given element of $\operatorname{USp}(L(\tau))$ has in general a multiplicity of bases of standard form. This apparently trivial fact has actually important consequences. 
From the boson algebra (3.6), it follows that for any element $W$ of $\operatorname{USp}(L(\tau))$, there may exist a state $|W\rangle_{b}$ such that for all $\xi \in W$,

$$
\begin{aligned}
& x(\bar{\xi})|W\rangle_{b}=0, \\
& \bar{x}(\xi)|W\rangle_{b}=0 .
\end{aligned}
$$

Further, when this is the case, this state is unique up to normalization in each irreducible representation of the algebra. The natural question arises about the conditions under which the above equations have a non-trivial solution. We first consider the simple case where $W$ is the subspace $W_{I}$ of $L(\tau)$ defined by a basis of the form (3.9a-b) with all the $R$ and $S$ matrices identically zero. It is easily seen that $W_{I}$ belongs to $\operatorname{USp}(L(\tau))$ and that the only basis of $W_{I}$ of standard form is that which defines it. The corresponding state $\left|W_{I}\right\rangle_{b}$ exists and is indeed the ground state $\left|\tau_{I}\right\rangle_{b}$ of an irreducible Fock representation of the boson algebra:

$$
\begin{array}{ll}
\alpha_{m-\tau_{j}}^{j}\left|\tau_{I}\right\rangle_{b}=0, & m \in I_{j}, \\
\tilde{\alpha}_{m+\tau_{j}}^{j}\left|\tau_{I}\right\rangle_{b}=0, & m \in-I_{j}, \\
\bar{\alpha}_{m+\tau_{j}}^{j}\left|\tau_{I}\right\rangle_{b}=0, & m \in-I_{j}, \\
\tilde{\tilde{\alpha}}_{m-\tau_{j}}^{j}\left|\tau_{I}\right\rangle_{b}=0, & m \in I_{j} .
\end{array}
$$

Note in particular that the state $\left|L_{+}\right\rangle_{b}$ is just the standard bosonic ground state $|\tau\rangle_{b}=\left|\tau_{I}\right\rangle_{b}$, where by $I_{j}=\mathbf{N}+\left[\tau_{j}\right]$ and $[x]$ denotes the integer part of a real number $x$. This fact justifies our choice of $L_{+}$as the reference maximal isotropic subspace. In this way, we see that there is a correspondence between the $h$-ples $I_{j}$ of sets of integers obeying the conditions stated earlier and the inequivalent irreducible Fock representations of the boson algebra. If $W$ is an arbitrary element of $\operatorname{USp}(L(\tau))$, then there is a non-trivial solution $|W\rangle_{I b}$ in the irreducible representation corresponding to a given $h$-ple $I_{j}$ if and only if $W$ has a basis of standard form with the same sets $I_{j}$. A straightforward albeit tedious algebraic calculation shows that

$$
|W\rangle_{I b}=\exp \left(B_{W I}\right)\left|\tau_{I}\right\rangle_{b}
$$

where

$$
\begin{aligned}
B_{W I}= & \frac{1}{2} \sum_{j=1}^{h} \sum_{k=1}^{h}\left[\sum_{r \in-I_{j}, s \in I_{k}} R_{r+\tau_{j}, s-\tau_{k}}^{j k} \frac{1}{r+\tau_{j}} \frac{1}{s-\tau_{k}} \alpha_{-r-\tau_{j}}^{j} \bar{\alpha}_{-s+\tau_{k}}^{k}\right. \\
& +\sum_{r \in-I_{j}, s \in \overline{-I}} S_{r+\tau_{j}, s+\tau_{k}}^{j k} \frac{1}{r+\tau_{j}} \frac{1}{s+\tau_{k}} \alpha_{-r-\tau_{j}}^{j} \tilde{\tilde{\alpha}}_{-s-\tau_{k}}^{k} \\
& +\sum_{r \in I_{j}, s \in \overline{-I_{k}}} \tilde{R}_{r-\tau_{j}, s+\tau_{k}}^{j k} \frac{1}{r-\tau_{j}} \frac{1}{s+\tau_{k}} \tilde{\alpha}_{-r+\tau_{j}}^{j} \tilde{\tilde{\alpha}}_{-s-\tau_{k}}^{k} \\
& \left.+\sum_{r \in I_{j}, s \in I_{k}} \tilde{S}_{r-\tau_{j}, s-\tau_{k}}^{j k} \frac{1}{r-\tau_{j}} \frac{1}{s-\tau_{k}} \tilde{\alpha}_{-r+\tau_{j}}^{j} \bar{\alpha}_{-s+\tau_{k}}^{k}\right] .
\end{aligned}
$$


The state $\left|\tau_{I}\right\rangle_{b}$ is related to $|\tau\rangle_{b}$ by

$$
\begin{aligned}
\left|\tau_{I}\right\rangle_{b}= & \prod_{j=1}^{h}\left[\prod_{m \in I_{j} \backslash\left(\mathbf{N} \cup\left\{1+\left[\tau_{j}\right]\right\}\right)} \delta\left(\alpha_{m-\tau_{j}}^{j}\right) \delta\left(\tilde{\bar{\alpha}}_{m-\tau_{j}}^{j}\right)\right] \\
& \cdot\left[\prod_{m \in-I_{j} \backslash\left(\mathrm{N} \cup\left\{-\left\{\tau_{j}\right]\right\}\right)} \delta\left(\tilde{\alpha}_{m+\tau_{j}}^{j}\right) \delta\left(\bar{\alpha}_{m+\tau_{j}}^{j}\right)\right]|\tau\rangle_{b} .
\end{aligned}
$$

The $\delta$ operators can be defined by bosonizing the $x-\bar{x}$ system or can be dealt with by means of an appropriate generalization of the Gaussian representation introduced in the third reference [6].

We now introduce an interesting generalization of the above construction. For any element $W$ of $\operatorname{USp}(L(\tau))$ and any element $\zeta$ of $L(\tau)$, we consider the state $|W+\zeta\rangle_{b}$ satisfying the equations

$$
\begin{aligned}
& x(\bar{\xi})|W+\zeta\rangle_{b}=\Phi_{b}(\zeta, \bar{\xi})|W+\zeta\rangle_{b}, \\
& \bar{x}(\xi)|W+\zeta\rangle_{b}=-\Phi_{b}(\xi, \bar{\zeta})|W+\zeta\rangle_{b} .
\end{aligned}
$$

Geometrically, $|W+\zeta\rangle_{b}$ corresponds to the hyperplane $W+\zeta$ of $L(\tau)$. This indicates a possible affine generalization of the $\operatorname{USp}(L(\tau))$ Grassmannian. The state $|W+\zeta\rangle_{b}$ exists in the bosonic Fock representation corresponding to $I$ whenever $|W\rangle_{b}$ does. In that case, we have the following relation:

$$
|W+\zeta\rangle_{I b}=\exp \left\{\frac{1}{2}[x(\bar{\zeta})+\bar{x}(\zeta)]\right\}|W\rangle_{I b} \text {. }
$$

Parenthetically, we note that when $\zeta$ belongs to $W_{I}^{\perp}$, the operator $x(\bar{\zeta})+\bar{x}(\zeta)$ commutes with $B_{W I}$ and annihilates the state $\left\langle\tau_{I}\right|$ (cfr. Eqs. $(3.11-12)$ ).

Next we consider the fermions. In this case the relevant algebraic structure is the twisted $U$-Grassmannian. The theory of $U$-Grassmannians can be formulated on the same lines as that of the USp-Grassmannians. So we shall limit overselves to provide the essential definitions and facts.

We define $M(\tau)$ as the space of the formal power series

$$
\begin{gathered}
\chi^{j}\left(z_{j}, \bar{z}_{j}\right)=\sum_{r=-\infty}^{\infty}\left[d z_{j}^{1 / 2} \chi_{r-\tau_{j}}^{j} z_{j}^{r-1 / 2-\tau_{j}}+d \bar{z}_{j}^{1 / 2} \tilde{\chi}_{r+\tau_{j}}^{j} \bar{z}_{j}^{r-1 / 2+\tau_{j}}\right], \\
\sum_{r=-\infty}^{\infty}\left[\left|\chi_{r-\tau_{j}}^{j}\right|^{2}+\left|\tilde{\chi}_{r+\tau_{j}}^{j}\right|^{2}\right]<\infty,
\end{gathered}
$$

where $r \in \mathbf{Z}+\frac{1}{2}$. As it will be explained later, this setting can be used to describe both twisted Neveu-Schwartz and Ramond fermions by a suitable definition of the twists $\tau$.

In analogy with the bosonic case, there is a natural non-singular hermitian sesquilinear form on $M(\tau)$ which makes it a unitary space. This is also related to the global $U(1)$ symmetry of the action $S_{f}[\psi, \tilde{\psi}]$,

$$
\Phi_{f}(\chi, \bar{\phi})=\sum_{j=1}^{h} \frac{1}{2 \pi i} \oint_{S^{1}} \chi^{j} \gamma^{3} \bar{\phi}^{j}=\sum_{j=1}^{h} \sum_{r=-\infty}^{\infty}\left[\chi_{r-\tau_{j}}^{j} \overline{\tilde{\phi}_{-r+\tau_{j}}^{j}}+\tilde{\chi}_{r+\tau_{j}}^{j} \overline{\phi_{-r-\tau_{j}}^{j}}\right]
$$

We denote by $U(M(\tau))$ the corresponding group of continuous unitary operators. 
Next we introduce a set of operators $\psi_{m-\tau_{j}}^{j}, \tilde{\psi}_{m+\tau_{j}}^{j}, \bar{\psi}_{m+\tau_{j}}^{j}$ and $\tilde{\bar{\psi}}_{m-\tau_{j}}^{j}$ obeying the anticommutation relations

$$
\begin{aligned}
& {\left[\psi_{m-\tau_{j}}^{j}, \bar{\psi}_{n+\tau_{k}}^{k}\right]_{+}=-2 \delta_{m+n, 0} \delta^{j k},} \\
& {\left[\tilde{\psi}_{m+\tau_{j}}^{j}, \tilde{\bar{\psi}}_{n-\tau_{k}}^{k}\right]_{+}=-2 \delta_{m+n, 0} \delta^{j k},}
\end{aligned}
$$

and the adjunction relations

$$
\begin{aligned}
& \psi_{m-\tau_{j}}^{j}=\bar{\psi}_{-m+\tau_{j}}^{j}, \\
& \tilde{\psi}_{m+\tau_{j}}^{j} \dagger=\widetilde{\bar{\psi}}_{-m-\tau_{j}}^{j}
\end{aligned}
$$

The oscillatory part of the fermionic field operators is given by

$$
\begin{aligned}
& \psi^{j}\left(z_{j}, \bar{z}_{j}\right)=\sum_{r=-\infty}^{\infty}\left[d z_{j}^{1 / 2} \psi_{r-\tau_{j}}^{j} z_{j}^{r-1 / 2-\tau_{j}}+d \bar{z}_{j}^{1 / 2} \tilde{\psi}_{r+\tau_{j}}^{j} \bar{z}_{j}^{r-1 / 2+\tau_{j}}\right], \\
& \bar{\psi}^{j}\left(z_{j}, \bar{z}_{j}\right)=\sum_{r=-\infty}^{\infty}\left[d z_{j}^{1 / 2} \bar{\psi}_{r+\tau_{j}}^{j} z_{j}^{r-1 / 2+\tau_{j}}+d \bar{z}_{j}^{1 / 2} \tilde{\bar{\psi}}_{r-\tau_{j}}^{j} \bar{z}_{j}^{r-1 / 2-\tau_{j}}\right] .
\end{aligned}
$$

For any $\chi$ in $M(\tau)$, we define the smeared operators

$$
\begin{aligned}
& \psi(\bar{\chi})=\Phi_{f}(\psi, \bar{\chi})=\sum_{j=1}^{h} \sum_{r=-\infty}^{\infty}\left[\overline{\tilde{\chi}_{r+\tau_{j}}^{j}} \psi_{-r-\tau_{j}}^{j}+\overline{\chi_{r-\tau_{j}}^{j}} \tilde{\psi}_{-r+\tau_{j}}^{j}\right] \\
& \bar{\psi}(\chi)=\Phi_{f}(\chi, \bar{\psi})=\sum_{j=1}^{h} \sum_{r=-\infty}^{\infty}\left[\chi_{r-\tau_{j}}^{j} \bar{\psi}_{-r+\tau_{j}}^{j}+\tilde{\chi}_{r+\tau_{j}}^{j} \tilde{\bar{\psi}}_{-r-\tau_{j}}^{j}\right] .
\end{aligned}
$$

The operators $\psi(\bar{\chi})$ and $\bar{\psi}(\chi)$ obey the algebra

$$
[\bar{\psi}(\chi), \psi(\bar{\phi})]_{+}=-2 \Phi_{f}(\chi, \bar{\phi}),
$$

the remaining anticommutators being zero.

In analogy to the bosonic case, the maximal anticommuting subalgebras of the algebra (3.20) correspond to the maximal isotropic subspaces of the unitary space $M(\tau)$. The manifold of these subspaces can be defined in terms of a given polarization

$$
M(\tau)=M_{+} \oplus M_{-},
$$

where $M_{ \pm}$are maximal isotropic subspaces of $M(\tau)$. Let $U_{\text {res }}(M(\tau))$ be the subgroup of $U(M(\tau))$ formed by all elements of $U(M(\tau))$ such that $U_{+-}$and $U_{-+}$are Hilbert-Schmidt operators. The twisted Grassmannian manifold $U(M(\tau))$ is the homogeneous space

$$
\mathbf{U}(M(\tau))=\frac{U_{\mathrm{res}}(M(\tau))}{\left[G L\left(M_{+}\right) \times G L\left(M_{-}\right)\right]_{\mu}},
$$

where the map $\mu$ is defined in a way similar to the bosonic case. In the present context, $M_{+}$is properly defined as the subspace of all $\chi \in M(\tau)$ such that $\chi_{m-\tau_{j}}^{j}=0$ for $m>\tau_{j}$ and $\tilde{\chi}_{m+\tau_{j}}^{j}=0$ for $m \geqq-\tau_{j}$.

From the fermion algebra (3.20), it follows that for any element $W$ of $\mathbf{U}(M(\tau))$ 
there is a state $|W\rangle_{f}$ such that for all $\chi \in W$,

$$
\begin{aligned}
& \psi(\bar{\chi})|W\rangle_{f}=0 \\
& \bar{\psi}(\chi)|W\rangle_{f}=0
\end{aligned}
$$

As is well-known, there is only one irreducible Fock representation of the fermion algebra. Thus, unlike the bosonic case, the state $|W\rangle_{f}$ always exists and is unique up to normalization. In particular, it is seen that the state $\left|M_{+}\right\rangle_{f}$ is just the standard fermionic ground state $|\tau\rangle_{f}$. The computation of $|W\rangle_{f}$ for a general element $W$ of $\mathbf{U}(M(\tau))$ is totally analogous to that of its bosonic counterpart. The straightforward algebraic details are left to the reader.

We now shall establish a correspondence which is the counterpart of the Krichever map in the present framework [15]. Following Vafa [6,16], we consider the manifold $\mathscr{P}(g, h)$ of all Riemann surfaces $\Sigma$ of genus $g$ with $h$ punctures and a choice of a local holomorphic coordinate $z_{k}$ at each puncture $p_{k}$ such that $z_{k}\left(p_{k}\right)=0$ and equipped with a twist structure $\gamma$ (cfr. Sect. 2). More generally, we may assign a holomorphic coordinate only to a subset of the punctures. In fact, in a twisted setting a given number of punctures must be included in any case in order to provide the twisted fields with the appropriate multivaluedness. However, it turns out that the above definition is sufficient for our purposes. We now shall show that for any element $\Sigma(\gamma) \in \mathscr{P}(g, h)$ there are associated two elements $W_{b}(\Sigma(\gamma))$ and $W_{f}(\Sigma(\gamma))$ of $\mathbf{U S p}(L(v(\gamma)))$ and $\mathbf{U}(M(v(\gamma)))$, respectively. In the following analysis we shall consider only special twist structures for simplicity. The generalization to general twist structures is straightforward.

We consider first the bosons. For a given $\Sigma(\gamma)$ in $\mathscr{P}(g, h)$, we consider the subspace $\mathscr{W}_{b}(\Sigma(\gamma))$ of $\Gamma\left(\Sigma_{c}, \mathscr{M}^{+}(1, \gamma) \oplus \overline{\left.\mathscr{M}^{-}(1, \gamma)\right)}\right.$ formed by all functions which are twisted harmonic off the punctures (see Sect. 2). Since $\Sigma(\gamma)$ is endowed with a choice of a coordinate at each puncture vanishing at the puncture, the restriction of $\mathscr{W}_{b}(\Sigma(\gamma))$ to the coordinate domains defines a subspace $W_{b}(\Sigma(\gamma))$ of $L(v(\gamma))$. By using the Green identity for the Laplacian operator on $\Sigma$, it is possible to show that $W_{b}(\Sigma(\gamma))$ is an isotropic subspace of $L(v(\gamma))$, which is easily seen to be maximal. However, in general $W_{b}(\Sigma(\gamma))$ does not belong to $\operatorname{USp}(L(v(\gamma)))$ as the condition (3.9f) fails to hold for any given basis of standard form. This is reminiscent of the occurrence of plane waves in Quantum Mechanics even though these are not physical wave packets.

A similar analysis can be carreid out for the fermions. In this case, for any element $\Sigma(\gamma)$ of $\mathscr{P}(g, h)$ one considers the subspace $\mathscr{W}_{f}(\Sigma(\gamma))$ of $\Gamma\left(\Sigma_{c}, \mathscr{M}^{+}(\lambda, \gamma)\right) \oplus$ $\overline{\Gamma\left(\Sigma_{c}, \mathscr{M}^{-}(\lambda, \gamma)\right)}$ consisting of all sections which either are (anti)holomorphic or have poles at the punctures and are (anti)holomorphic everywhere else. Here $\lambda$ is one of the spin bundles of $\Sigma_{c}$. As in the bosonic case, $\mathscr{W}_{f}(\Sigma(\gamma))$ defines a maximal isotropic subspace $W_{f}(\Sigma(\gamma))$ of $M(v(\gamma))$.

From the above discussion and from Grassmannian theory, we see that to any Riemann surface $\Sigma(\gamma)$ in $\mathscr{P}(g, h)$ we can associate two states in the appropriate Fock representations, namely $\left|W_{b}(\Sigma(\gamma))\right\rangle_{I b}$ and $\left|W_{f}(\Sigma(\gamma))\right\rangle_{f}$. Note that for a given Riemann surface $\Sigma(\gamma)$, there are in general several states $\left|W_{b}(\Sigma(\gamma))\right\rangle_{I b}$ belonging to inequivalent irreducible Fock representations of the boson algebra. These 
correspond to different pictures, in analogy to the bosonic ghosts [17]. The origin of this multiplicity is not simply algebraic but can be traced back to the structure of the space $\mathscr{W}_{b}(\Sigma(\gamma))$ and thus to the geometry of $\Sigma(\gamma)$.

As in the standard case, in general not all elements of $\mathscr{W}_{b}(\Sigma(\gamma))$ can be expressed as a sum of a twisted meromorphic and antimeromorphic section, as the twisted Weierstrass gap theorem imposes restrictions on the order of the poles of the twisted (anti)meromorphic functions. As a consequence, the $S$ and $\tilde{S}$ matrices (cfr. Eqs. (3.9a-f)) are non-zero and the state $\left|W_{b}(\Sigma(\gamma))\right\rangle_{1 b}$ cannot be expressed as a tensor product of a left- and right moving part [6]. Conversely, it is always possible to choose a chiral basis for $\mathscr{W}_{f}(\Sigma(\gamma))$ and $\left|W_{f}(\Sigma(\gamma))\right\rangle_{f}$ factorizes in left- and right moving components.

The basic problem of the operator formalism is the computation of the states $\left|W_{b}(\Sigma(\gamma))\right\rangle_{I b}$ and $\left|W_{f}(\Sigma(\gamma))\right\rangle_{f}$. In practice, we need to compute these states only for the cases where $\Sigma$ is a sphere with two or three punctures, i.e. the sewing and the vertex states. By means of the sewing operation, we can compute states for higher values of $g$ and $h$ in terms of these two basic states [6]. In the bosonic case, of course, we have to make sure that the representations of the boson algebra involved match so that the result does not vanish identically.

As an illustration of the above results, we consider the sewing state. We parametrize the Riemann sphere with the usual complex coordinate $z$ and assume that the punctures are located at $z=0$ and $z=\infty$ with coordinates $z_{1}=z$ and $z_{2}=1 / z$, respectively. We further assume for simplicity that the twists of both punctures are non-zero. For any integer $q$ there is a chiral basis of standard form of $W_{b}\left(\Sigma_{0,2}\left(\gamma_{2}\right)\right)$, namely $\eta_{-m-v_{1}}^{1}(z, \bar{z})=z^{-m-v_{1}}, m \geqq-q-\left[v_{1}\right], \eta_{-m-v_{2}}^{2}(z, \bar{z})=z^{m+v_{2}}$, $m \geqq q-\left[v_{2}\right], \tilde{\eta}_{-m+v_{1}}^{1}(z, \bar{z})=\bar{z}^{-m+v_{1}}, m>q+\left[v_{1}\right]$ and $\tilde{\eta}_{-m+v_{2}}^{2}(z, \bar{z})=\bar{z}^{m^{2} v_{2}}, m>-q+$ $\left[v_{2}\right]$, where $v_{j}=v_{j}(\gamma)$. The corresponding state is

$$
\begin{aligned}
\left|W_{b}\left(\Sigma_{0,2}\left(\gamma_{2}\right)\right)\right\rangle_{q b}= & \exp \left[\frac{1}{2} \sum_{r=-q-\left[v_{1}\right]}^{\infty} \frac{1}{r+v_{1}}\left(\alpha_{-r-v_{1}}^{1} \bar{\alpha}_{-r-v_{1}}^{2}+\tilde{\alpha}_{-r-v_{1}}^{2} \tilde{\bar{\alpha}}_{-r-v_{1}}^{1}\right)\right. \\
& \left.+\frac{1}{2} \sum_{r=q-\left[v_{2}\right]}^{\infty} \frac{1}{r+v_{2}}\left(\alpha_{-r-v_{2}}^{2} \bar{\alpha}_{-r-v_{2}}^{1}+\tilde{\alpha}_{-r-v_{2}}^{1} \tilde{\bar{\alpha}}_{-r-v_{2}}^{2}\right)\right]|v\rangle_{q b} .
\end{aligned}
$$

It is possible to compute also the vertex state even though the resulting expressions are rather unwiedly and not particularly illuminating.

Similar calculations can be carried out for the fermions. Ther fermionic sewing state has a particularly simple expression

$$
\begin{aligned}
\left|W_{f}\left(\Sigma_{0,2}\left(\gamma_{2}\right)\right)\right\rangle_{f}= & \exp \left[\frac { \pm i } { 2 } \sum _ { r = 1 / 2 } ^ { \infty } \left(\psi_{-r+v_{2}}^{1} \bar{\psi}_{-r+v_{2}}^{2}-\psi_{-r-v_{2}}^{2} \bar{\psi}_{-r-v_{2}}^{1}\right.\right. \\
& \left.\left.-\tilde{\psi}_{-r-v_{2}}^{1} \tilde{\bar{\psi}}_{-r-v_{2}}^{2}+\tilde{\psi}_{-r+v_{2}}^{2} \tilde{\bar{\psi}}_{-r+v_{2}}^{1}\right)\right]|v\rangle_{f},
\end{aligned}
$$

where $v_{j}=v_{j}(\gamma)$ and we assume that $v_{1}, v_{2} \neq-1 / 2,0$ and $v_{2}>0$ for simplicity. The sign ambiguity reflects the two choices of the relative sign of $d z_{1}^{1 / 2}$ and $d z_{2}^{1 / 2}$.

It is possible to find semiexplicit formulae for the state $\left|W_{b}(\Sigma(\gamma))\right\rangle_{I b}$ in the 
standard representation $I_{j}=\mathbf{N}+\left[v_{j}(\gamma)\right]$. For convenience, we parametrize the twists as follows:

$$
\mu_{l}(\gamma)=v_{l}(\gamma)-\left[v_{l}(\gamma)\right]
$$

Note that $0 \leqq \mu_{l}(\gamma)<1$. This is in fact the customary parametrization of the twists found in the literature $[4,7]$. Then it can be seen that

$$
\begin{array}{ll}
R_{r+\mu_{i}, s-\mu_{j}}^{i j}=\left.\frac{2\left(r+\mu_{i}\right)\left(s-\mu_{j}\right)}{r ! s !} \partial_{w_{i}}^{r} \partial_{z_{j}}^{s} w_{i}^{-\mu_{i}} z_{j}^{\mu_{J}} K(w, \bar{w} \mid z, \bar{z})\right|_{w=z=0}, & r \geqq 0, s>0 \\
S_{r+\mu_{i}, s+\mu_{j}}^{i j}=\left.\frac{2\left(r+\mu_{i}\right)\left(s+\mu_{j}\right)}{r ! s !} \partial_{w_{l}}^{r} \bar{\partial}_{z_{j}}^{s} w_{i}^{-\mu_{i}} \bar{z}_{j}^{-\mu_{j}} K(w, \bar{w} \mid z, \bar{z})\right|_{w=z=0}, & r \geqq 0, s \geqq 0 \\
\tilde{R}_{r-\mu_{i}, s+\mu_{j}}^{i j}=\left.\frac{2\left(r-\mu_{i}\right)\left(s+\mu_{j}\right)}{r ! s !} \bar{\partial}_{w_{i}}^{r} \bar{\partial}_{z_{j}}^{s} \bar{w}_{i}^{\mu_{i}} \bar{z}_{j}^{-\mu_{j}} K(w, \bar{w} \mid z, \bar{z})\right|_{w=z=0}, & r>0, s \geqq 0 \\
\tilde{S}_{r-\mu_{i}, s-\mu_{J}}^{i j}=\left.\frac{2\left(r-\mu_{i}\right)\left(s-\mu_{j}\right)}{r ! s !} \bar{\partial}_{w_{i}}^{r} \partial_{z_{j}}^{s} \bar{w}_{i}^{\mu_{i}} z_{j}^{\mu_{j}} K(w, \bar{w} \mid z, \bar{z})\right|_{w=z=0}, & r>0, s>0
\end{array}
$$

where we have suppressed the $\gamma$ dependence for simplicity. The kernel $K(w, \bar{w} \mid z, \bar{z})$ can be computed in terms of the suitably regularized two point correlators of the derivatives of the fields $x$ and $\bar{x}$ on $\Sigma$ :

$$
K(w, \bar{w} \mid z, \bar{z})=\frac{1}{4} \sum_{i, j=1}^{h} k\left(w_{i}, \bar{w}_{i} \mid z_{j}, \bar{z}_{j}\right),
$$

where, in terms of the local coordinates of the $i^{\text {th }}$ and $j^{\text {th }}$ puncture

$$
\begin{aligned}
k\left(w_{i}, \bar{w}_{i} \mid z_{j}, \bar{z}_{j}\right)= & \int_{0}^{w_{i}} d u_{i} \int_{0}^{z_{j}} d v_{j} \mathscr{R}\left\langle\partial x\left(u_{i}\right) \partial \bar{x}\left(v_{j}\right)\right\rangle \\
& +\int_{0}^{w_{i}} d \bar{u}_{i} \int_{0}^{z_{j}} d v_{j}\left\langle\bar{\partial} x\left(\bar{u}_{i}\right) \partial \bar{x}\left(v_{j}\right)\right\rangle \\
& +\int_{0}^{w_{i}} d \bar{u}_{i} \int_{0}^{z_{j}} d \bar{v}_{j} \mathscr{R}\left\langle\bar{\partial} x\left(\bar{u}_{i}\right) \bar{\partial} \bar{x}\left(\bar{v}_{j}\right)\right\rangle \\
& +\int_{0}^{w_{i}} d u_{i} \int_{0}^{z_{j}} d \bar{v}_{j}\left\langle\partial x\left(u_{i}\right) \bar{\partial} \bar{x}\left(\bar{v}_{j}\right)\right\rangle,
\end{aligned}
$$

and the correlators are regularized as follows:

$$
\begin{aligned}
& \mathscr{R}\left\langle\partial x\left(u_{i}\right) \partial \bar{x}\left(v_{j}\right)\right\rangle=\left\langle\partial x\left(u_{i}\right) \partial \bar{x}\left(v_{j}\right)\right\rangle+2 u_{i}^{-\mu_{i}} v_{j}^{1-\mu_{j}} \frac{\mu_{i} u_{i}+\left(1-\mu_{j}\right) v_{j}}{\left(u_{i}-v_{j}\right)^{2}} \delta_{i j} \\
& \mathscr{R}\left\langle\bar{\partial} x\left(\bar{u}_{i}\right) \bar{\partial} \bar{x}\left(\bar{v}_{j}\right)\right\rangle=\left\langle\bar{\partial} x\left(\bar{u}_{i}\right) \bar{\partial} \bar{x}\left(\bar{v}_{j}\right)\right\rangle+2 \bar{u}_{i}^{-\mu_{j}} \bar{v}_{j}^{1-\mu_{j}} \frac{\mu_{i} \bar{u}_{i}+\left(1-\mu_{j}\right) \bar{v}_{j}}{\left(\bar{u}_{i}-\bar{v}_{j}\right)^{2}} \delta_{i j} .
\end{aligned}
$$

The above correlators have been computed in terms of theta functions in ref. [12]. The regulator of the operator product singularity of the correlators $\left\langle\partial x\left(u_{i}\right) \partial \bar{x}\left(v_{j}\right)\right\rangle$ and $\left\langle\bar{\partial} x\left(\bar{u}_{i}\right) \bar{\partial} \bar{x}\left(\bar{v}_{j}\right)\right\rangle$ has the same analytic form of the corresponding correlators on the sphere, but, of course, is restricted to the appropriate domains of the relevant 
variables. The choice of 0 as base point of the integration path is the only one which provides the correct monodromy at the $i^{\text {th }}$ and $j^{\text {th }}$ puncture.

A similar analysis can be carried out for the fermions. In this case, however, the possible existence of zero modes associated with twisting introduces an element of complication. However, when $n(\gamma)=0$ (cfr. Eq. (2.1)) there are no zero modes in the generic situation, by the twisted Riemann-Roch theorem (cfr. Eqs. (2.6-7)). In this case $\left|W_{f}(\Sigma(\gamma))\right\rangle_{f}$ can be computed in terms of the derivatives of the suitably regularized two point correlators of the fermion fields as in the bosonic case. The analysis is completely analogous to the preceding paragraph and is not expounded for brevity.

\section{Orbifold Operator Formalism}

We now shall apply the formalism developed in the previous sections to orbifold string models.

We consider an abelian orbifold $\Omega$ with point group $P$, lattice $L$, space group $S$ and base space $E$ [4]. Since $P$ is an abelian subgroup of $O(E), E_{\mathbf{C}}$ decomposes into the direct sum $E_{\mathrm{C}}=E_{1} \oplus E_{2} \oplus \cdots \oplus E_{d / 2} \oplus \bar{E}_{1} \oplus \bar{E}_{2} \cdots \oplus E_{d / 2}$, where the $E_{a}$ 's are 1-dimensional subspaces of $E_{\mathbf{C}}$ invariant under $P$.

A point group structure on a Riemann surface $\Sigma$ is defined analogously to a twist structure, by replacing $\Gamma$ by $P$. It is clear that the restriction of $P$ to $E_{a}$ form a twist group $\Gamma_{a}$ and that any point group structure $\pi$ determines a $\Gamma_{a}$ twist structure $\gamma_{a}(\pi)$ for each $a=1, \ldots, d / 2$.

When dealing with fermions, we have to account not only for the standard orbifold twists but also for the spin twists. The spin twist group is clearly $\mathbf{Z}_{2}$. Therefore, while the bosonic twist group for the $a^{t h}$ complex dimension is $\Gamma_{a}$ the fermionic twist group is properly $\mathbf{Z}_{2} \times \Gamma_{a}$. Note further that the combination of a spin twist $\sigma$ and an orbifold twist $v_{a}$ is the twist $\sigma+v_{a}-\left[\sigma+v_{a}+\frac{1}{2}\right]$. Spin twists around the punctures of a given Riemann surface are described by $\mathbf{Z}_{2}$ twist structures. For a given $\mathbf{Z}_{2}$ twist structure $\alpha$, the twist $\sigma_{j}(\alpha)$ around the $j^{\text {th }}$ puncture determines the fermionic type of that puncture: the puncture is Neveu-Schwartz if $\sigma_{j}(\alpha)=0$ and Ramond if $\sigma_{j}(\alpha)=-1 / 2$ [6].

A space group structure describes the multivaluedness of world-sheet instanton solutions on a given Riemann surface $\Sigma$. A space group structure can be viewed as a homomorphism of the first homotopy group $\pi_{1}(\Sigma)$ into the space group $S$. Two space group structures related by the adjoint action of $S$ are physically equivalent. Thus, the set of the inequivalent space group structures is properly $\operatorname{Hom}\left(\pi_{1}(\Sigma), S\right) / \operatorname{Ad} S$. To any space group structure $\omega$ there is naturally associated a unique point group structure $\pi_{\omega}$ by means of the natural projection $S \mapsto S / L \equiv P$. For brevity, we shall write $\gamma_{a}(\omega)$ instead of $\gamma_{a}\left(\pi_{\omega}\right)$.

To describe the conformal field theory associated to an orbifold model, we need the following data: i) a Riemann surface of genus $g$ and $h$ punctures with a choice of a holomorphic coordinate at each puncture vanishing at the puncture, ii) a $\mathbf{Z}_{2}$ twist structure $\alpha$ and iii) a space group structure $\omega$. Any orbifold correlation function factorizes into a quantum and instanton contribution

$$
\langle\cdots\rangle=\sum\langle\cdots\rangle_{q u} \cdot\langle\rangle_{c l}
$$


A quantum correlation function involving a product of local fields defined in the coordinate domains of $\Sigma$ can be written as

$$
\langle\cdots\rangle_{q u}={ }_{q u}\langle\alpha, \omega|\cdots| \Sigma, \alpha, \omega\rangle_{q u} .
$$

Here

$$
|\Sigma, \alpha, \omega\rangle_{q u}=|\Sigma, \alpha\rangle_{\text {ghosts }} \otimes|\Sigma, \alpha, \omega\rangle_{\text {matter }} .
$$

$|\Sigma, \alpha\rangle_{\text {ghosts }}$ is the state associated to the Faddeev-Popov (super) ghosts with a choice of picture compatible with the $\mathbf{Z}_{2}$ twist structure $\alpha$ and has been exhaustively described in the third reference [6]. Similarly, $|\Sigma, \alpha, \omega\rangle_{\text {matter }}$ is the state associated to the matter sector and is given by

$$
|\Sigma, \alpha, \omega\rangle_{\text {matter }}=\bigotimes_{a=1}^{d / 2}\left|W_{b}\left(\Sigma\left(\gamma_{a}(\omega)\right)\right)\right\rangle_{I b} \otimes\left|W_{f}\left(\Sigma\left(\alpha \times \gamma_{a}(\omega)\right)\right)\right\rangle_{f},
$$

where the states $\left|W_{b}(\Sigma(\gamma))\right\rangle_{I b}$ and $\left|W_{f}(\Sigma(\gamma))\right\rangle_{f}$ have been defined in Sect. (3) (cfr. Eqs. (3.24)). Finally,

$$
|\alpha, \omega\rangle_{q u}=|\alpha\rangle_{\text {ghosts }} \otimes \bigotimes_{a=1}^{d / 2}\left|v\left(\gamma_{a}(\omega)\right)\right\rangle_{I b} \otimes\left|v\left(\alpha \times \gamma_{a}(\omega)\right)\right\rangle_{f} .
$$

where $|\alpha\rangle_{\text {ghosts }}$ is the appropriate ghost vacuum. Note that the ghost picture and bosonic representation of the states $|\Sigma, \alpha, \omega\rangle_{q u}$ and $|\alpha, \omega\rangle_{q u}$ must coincide in order to yield a non-vanishing result. Note further that the choice of a picture or a representation affects the type of operators used in the ghost and matter part of the correlators for a given amplitude. From the functional integral interpretation of the formalism, it is clear that the final result cannot depend on these choices. finally, we observe that when $\gamma_{a}(\omega)=1$, it is necessary to tensor the states $|\Sigma, \alpha, \omega\rangle_{q u}$ and $|\alpha, \omega\rangle_{q u}$ by an appropriate superposition of $h$-particle zero momentum states, as explained in ref. [6].

The state $|\Sigma, \alpha, \omega\rangle_{q u}$ contains all the dependence of the quantum correlation functions on the moduli of $\Sigma$ and the location of the twist and spin-twist field insertions. As explained in ref. [6] this dependence is determined by the action of the energy-momentum tensor on the state. This also fixes the relative normalization of states corresponding to different values of these parameters.

Next, we turn to the instanton part of the correlators. In the following analysis we shall use the convenient standard bosonic representation introduced at the end of the previous section (cfr. Eqs. (3.26-28)) and suppress the index $I$ throughout. We shall also assume for simplicity that all the twist structures involved are special.

For each element $\omega$ of $\operatorname{Hom}\left(\pi_{1}(\Sigma), S\right) / \operatorname{Ad} S$ there exists a world sheet instanton solution $y^{a}(\omega)$. The instanton contribution of the amplitudes is of the form

$$
\langle\cdots\rangle_{c l}=\sum_{\omega \in \Delta} \exp \left(-\sum_{a=1}^{d / 2} S_{b}\left[y^{a}(\omega)\right]\right) \cdots .
$$

Here $\Delta$ is a subset of $\operatorname{Hom}\left(\pi_{1}(\Sigma), S\right) / \operatorname{Ad} S$ depending on the amplitude under consideration. $S_{b}\left[y^{a}(\omega)\right]$ is just the action of the world sheet instanton $y^{a}(\omega)$. The ellipses represent insertions of the derivatives of $y^{a}(\omega)$ or $\bar{y}^{a}(\omega)$. These are given by 
the expression

$$
d y^{a}(\omega)=\sum_{\alpha=1}^{m_{a}(\omega)} X_{\alpha}^{a}(\omega) \kappa_{\alpha}^{a}(\omega)
$$

where the $\kappa_{\alpha}^{a}(\omega)$ 's are a basis of the space $K^{+}\left(\Sigma, \gamma_{a}(\omega)\right)$ (cfr. Eq. (2.12)), $m_{a}(\omega)$ is its dimension and the $X_{\alpha}^{a}(\omega)$ 's are $\omega$ dependent coefficients (see ref. [12] for details).

The incorporation of the instanton part of the correlators in the operator formalism is achieved by inserting a suitable operator in the quantum correlator,

$$
\langle\cdots\rangle=\left\langle\cdots \sum_{\omega \in \Delta a=1} \bigotimes^{d / 2} U^{a}(\omega)\right\rangle_{q u}=\left\langle\alpha, \omega\left|\cdots \sum_{\omega \in \Delta a} \bigotimes_{q=1}^{d / 2} U^{a}(\omega)\right| \Sigma, \alpha, \omega\right\rangle_{q u} .
$$

The operator $U^{a}(\omega)$ is given by

$$
\begin{aligned}
U^{a}(\omega)= & \exp \left\{-S_{b}\left[y^{a}(\omega)\right]-\frac{1}{4 \pi i} \sum_{j=1}^{h} \oint_{S^{1}}\left[x_{h}^{a j}(\omega) \partial_{j} \bar{y}^{a j}(\omega)-x_{a}^{a j}(\omega) \bar{\partial}_{j} \bar{y}^{a j}(\omega)\right.\right. \\
& \left.\left.+\bar{x}_{h}^{a j}(\omega) \partial_{j} y^{a j}(\omega)-\bar{x}_{a}^{a j}(\omega) \bar{\partial}_{j} y^{a j}(\omega)\right]\right\}
\end{aligned}
$$

where $x_{h, a}^{a j}(\omega)$ and $\bar{x}_{h, a}^{a j}(\omega)$ are the holomorphic and antiholomorphic parts of the operators $x^{a j}(\omega)$ and $\bar{x}^{a j}(\omega)$ given by (3.4) with $\tau=\gamma_{a}(\omega)$ and $y^{a j}(\omega)$ and $\bar{y}^{a j}(\omega)$ are the restrictions of $y^{a}(\omega)$ and $\bar{y}^{a}(\omega)$ to the coordinate domain of the $j^{\text {th }}$ puncture, respectively. Inserting $U^{a}(\omega)$ into the quantum correlators shifts the derivatives of the operators $x^{a}$ and $\bar{x}^{a}$ by the corresponding derivatives of the world sheet instantons $y^{a}(\omega)$ and $\bar{y}^{a}(\omega)$ and in this way reproduces correctly the combination of the quantum and instanton part of the total correlator. Let $\zeta_{a}^{a}(\omega)$ be the element of $L\left(v\left(\gamma_{a}(\omega)\right)\right)$ defined by the condition that $d_{j} \zeta_{\alpha}^{a j}(\omega)=\kappa_{\alpha}^{a j}(\omega)$, where $\kappa_{\alpha}^{a j}(\omega)$ is the restriction of $\kappa_{\alpha}^{a}(\omega)$ to the coordinate domain of the $j^{\text {th }}$ puncture. Then

$U^{a}(\omega)=\exp \left\{-S_{b}\left[y^{a}(\omega)\right]+\frac{1}{2} \sum_{\alpha=1}^{m_{a}(\omega)}\left[X_{\alpha}^{a}(\omega) \bar{x}^{a}(\omega)\left(\zeta_{\alpha}^{a}(\omega)\right)+\bar{X}_{\alpha}^{a}(\omega) x^{a}(\omega)\left(\overline{\zeta_{\alpha}^{a}}(\omega)\right)\right]\right\}$.

From (4.6), we see that the incorporation of the instanton $y^{a}(\omega)$ amounts to the replacement $\left|W_{b}\left(\Sigma\left(\gamma_{a}(\omega)\right)\right)\right\rangle_{b} \rightarrow\left|W_{b}\left(\Sigma\left(\gamma_{a}(\omega)\right)\right)+\sum_{\alpha=1}^{m_{a}(\omega)} X_{\alpha}^{a}(\omega) \zeta_{\alpha}^{a}(\omega)\right\rangle_{b}$ in Eq. (4.2c) (cfr. Eqs. (3.13-14)). In this way, the total amplitude can be associated to a family of hyperplanes in $L\left(v\left(\gamma_{a}(\omega)\right)\right)$ parallel to $W_{b}\left(\Sigma\left(\gamma_{a}(\omega)\right)\right)$ each of which corresponds to a particular instanton solution. We shall now provide an interpretation of the operators appearing in Eq. (4.6). In the following analysis we have been inspired by ref. [9]. To simplify the notation, we shall suppress the $a$ - and $\omega$-dependence which is not essential to our argument.

In Sect. 2 we have seen that the space of loops of zero $(\bmod Z)$ total twist is the dual space $K^{+}(\Sigma, \gamma)^{\vee}$ of the space of twisted harmonic forms $K^{+}(\Sigma, \gamma)$, the duality being defined by Eq. (2.13). Now we shall show the isomorphism

$$
K^{+}(\Sigma, \gamma)^{\vee} \equiv \overline{K_{n m}^{+}(\Sigma, \gamma) / K^{+}(\Sigma, \gamma)},
$$


where the $n_{l}$ 's and $m_{l}$ 's are non-negative integers such that

$$
\begin{aligned}
& \sum_{l=1}^{h} n_{l}=-n(\gamma)-\sum_{l=1}^{h}\left[-v_{l}(\gamma)\right]+g-1, \\
& \sum_{l=1}^{h} m_{l}=+n(\gamma)-\sum_{l=1}^{h}\left[+v_{l}(\gamma)\right]+g-1
\end{aligned}
$$

for $\gamma \neq 1$ and

$$
\sum_{l=1}^{h} n_{l}=\sum_{l=1}^{h} m_{l}=g
$$

otherwise. In fact, consider the sesquilinear pairing

$$
\begin{aligned}
f(\lambda, \kappa) & =\frac{1}{4 \pi i} \sum_{j=1}^{h} \oint_{S^{1}}\left[\bar{\lambda}_{a}^{j} \zeta_{h}^{j}(\kappa)-\bar{\lambda}_{h}^{j} \zeta_{a}^{j}(\kappa)\right] \\
& =\frac{1}{4 \pi i} \sum_{j=1}^{h} \oint_{S^{1}}\left[\kappa_{a}^{j} \bar{\zeta}_{h}^{j}(\lambda)-\kappa_{h}^{j} \bar{\zeta}_{a}^{j}(\lambda)\right]
\end{aligned}
$$

where $\lambda \in K_{n m}^{+}(\Sigma, \gamma), \kappa \in K^{+}(\Sigma, \gamma), \lambda^{j}$ and $\kappa^{j}$ are the restrictions of $\lambda$ and $\kappa$ to the coordinate domain of the $j^{\text {th }}$ puncture and $\zeta^{j}(\lambda)$ and $\zeta^{j}(\kappa)$ are defined by the condition $d_{j} \zeta^{j}(\lambda)=\lambda^{j}$ and $d_{j} \zeta^{j}(\kappa)=\kappa^{j}$, respectively. From (4.7d) and the definition of $K^{+}(\Sigma, \gamma)$, it is easy to see that the value of $f(\lambda, \kappa)$ is left unchanged by shifting $\lambda$ by any element of $K^{+}(\Sigma, \gamma)$. This is sufficient to prove the aforementioned isomorphism. From (4.7a), it follows that we can represent any loop of zero total twist as a differential of $K_{n m}^{+}(\Sigma, \gamma) \bmod K^{+}(\Sigma, \gamma)$. From this remark, (2.13), (3.2) and (4.7d), it follows fruther that to any loop $\lambda$ in $K^{+}(\Sigma, \gamma)^{\vee}$ and any form $\kappa$ in $K^{+}(\Sigma, \gamma)$ we can associate elements $\zeta(\lambda)$ and $\zeta(\kappa)$ of $L(v(\gamma))$ such that

$$
\begin{aligned}
& \Phi_{b}(\zeta(\kappa), \bar{\zeta}(\lambda))=\frac{1}{2}\langle\lambda, \kappa\rangle, \\
& \Phi_{b}\left(\zeta(\kappa), \bar{\zeta}\left(\kappa^{\prime}\right)\right)=0, \\
& \Phi_{b}\left(\zeta(\lambda), \bar{\zeta}\left(\lambda^{\prime}\right)\right)=0 .
\end{aligned}
$$

for $\lambda, \lambda^{\prime} \in K^{+}(\Sigma, \gamma)^{\vee}$ and $\kappa, \kappa^{\prime} \in K^{+}(\Sigma, \gamma)$. Define the operators

$$
\begin{aligned}
& a(\lambda)=x(\bar{\zeta}(\lambda)), \\
& \bar{a}(\lambda)=\bar{x}(\zeta(\lambda)), \\
& b(\kappa)=x(\bar{\zeta}(\kappa)), \\
& \bar{b}(\kappa)=\bar{x}(\zeta(\kappa)) .
\end{aligned}
$$

The form a finite dimensional subalgebra of the bosonic algebra (3.6) with the following commutation relations:

$$
\begin{aligned}
& {[a(\lambda), \bar{b}(\kappa)]=\langle\lambda, \kappa\rangle,} \\
& {[b(\kappa), \bar{a}(\lambda)]=-\langle\lambda, \kappa\rangle .}
\end{aligned}
$$

The operators $a(\lambda), \bar{a}(\lambda), b(\kappa)$ and $\bar{b}(\kappa)$ define a quantum system with finitely many 
degrees of freedom. The $a(\lambda)$ 's and $\bar{a}(\lambda)$ 's may be interpreted as momenta flowing through the loops of zero total twist and the $b(\kappa)$ 's and $\bar{b}(\kappa)$ 's as the corresponding canonically conjugate variables. In this case we have two types of variables corresponding to barred and unbarred quantities. A complete set of commuting operators is given by the $b(\kappa)$ 's and the $\bar{b}(\kappa)$ 's. This is particularly convenient in the present context as it is formed exactly by the operators appearing in Eq. (4.6). In this respect, our construction differs from that of ref. [9] in which a complete set of commuting operators was chosen corresponding to certain linear combinations of our $a$ 's and $b$ 's. Finally, we note that it is always possible to choose a basis of $K^{+}(\Sigma, \gamma)$ formed by differentials which are either holomorphic or antiholomorphic. In this way, the operators $b(\kappa)$ and $\bar{b}(\kappa)$ can be divided naturally in two chiral subsets.

One of the most attractive features of the standard operator formalism is that the states corresponding to Riemann surfaces of arbitrary genus and number of punctures can be obtained by sewing in all possible ways the states corresponding to the sphere with two and three punctures. Moreover, the result does not depend on the order in which these operations are performed. We wish to study sewing in the context of the orbifold operator formalism. Clearly, in the present case we have to account for the space group structures.

Consider the manifold $\mathscr{2}(g, h)$ of all Riemann surfaces $\Sigma(\alpha, \omega)$ endowed with the following structure: $i$ ) a choice of a holomorphic coordinate at each puncture vanishing at the puncture, ii) a spin structure $\alpha$ and iii) a space group structure $\omega$ $[6,16]$.

Vafa's operations ${ }_{j} \infty_{k}$ and $8_{k}^{j}$ can be generalized to the present setting. Let $\Sigma_{1}\left(\alpha_{1}, \omega_{1}\right)$ and $\Sigma_{2}\left(\alpha_{2}, \omega_{2}\right)$ be Riemann surfaces in $2\left(g_{1}, h_{1}\right)$ and $2\left(g_{2}, h_{2}\right)$, respectively. Let $p_{1 j}$ and $p_{2 k}$ be punctures of $\Sigma_{1}$ and $\Sigma_{2}$. Assume further that $\alpha_{1}\left(l_{1 j}\right) \cdot \alpha_{2}\left(l_{2 k}\right)=1$ and $\omega_{1}\left(l_{1 j}\right) \cdot \omega_{2}\left(l_{2 k}\right)=1$, where $l_{1 j}$ is the homology loop which only winds once around $p_{1 j}$ with the appropriate orientation and similarly $l_{2 k}$. We define a Riemann surface $\Sigma_{3}\left(\alpha_{3}, \omega_{3}\right)=\Sigma_{1}\left(\alpha_{1}, \omega_{1}\right)_{j} \infty_{k} \Sigma_{2}\left(\alpha_{2}, \omega_{2}\right)$ in $\mathscr{2}\left(g_{1}+g_{2}\right.$, $\left.h_{1}+h_{2}-2\right)$ as follows. $\Sigma_{3}$ is obtained by sewing the puncture $p_{1 j}$ of $\Sigma_{1}$ and $p_{2 k}$ of $\Sigma_{2}$ by using the plumbing fixture with parameter 1 (this is possible because we have assigned local holomorphic coordinates at the punctures). The resulting Riemann surface has clearly genus $g_{1}+g_{2}$ and $h_{1}+h_{2}-2$ punctures with a holomorphic coordinate at each puncture. Further, the above condition on $\alpha_{1}$ and $\alpha_{2}$ and $\omega_{1}$ and $\omega_{2}$ ensures that they are restrictions of a well-defined spin structure $\alpha_{3}$ and space group structure $\omega_{3}$ on $\Sigma_{3}$. This completely defines $\Sigma_{3}\left(\gamma_{3}\right)$. The operation $8_{k}^{j}$ is defined similarly.

To any element $\Sigma(\alpha, \omega)$ of $\mathscr{2}(g, h)$ we associate the state

$$
|\Sigma(\alpha, \omega)\rangle=\bigotimes_{a=1}^{d / 2} U^{a}(\omega)|\Sigma, \alpha, \omega\rangle_{q u} .
$$

Then, the state $\left|\Sigma_{1}\left(\alpha_{1}, \omega_{1}\right)_{j} \infty_{k} \Sigma_{2}\left(\alpha_{2}, \omega_{2}\right)\right\rangle$ is given in terms of the states $\left|\Sigma_{1}\left(\alpha_{1}, \omega_{1}\right)\right\rangle$ and $\left|\Sigma_{2}\left(\alpha_{2}, \omega_{2}\right)\right\rangle$ by

$$
\left|\Sigma_{1}\left(\alpha_{1}, \omega_{1}\right)_{j} \infty_{k} \Sigma_{2}\left(\alpha_{2}, \omega_{2}\right)\right\rangle=\left\langle S_{j k}|| \Sigma_{1}\left(\alpha_{1}, \omega_{1}\right)\right\rangle \otimes\left|\Sigma_{2}\left(\alpha_{2}, \omega_{2}\right)\right\rangle .
$$

Here, $\left|S_{j k}\right\rangle$ is the appropriate sewing state. Its spin and orbifold twists must match those of the punctures of $\Sigma_{1}$ and $\Sigma_{2}$ undergoing sewing. 


\section{Discussion and conclusions}

In this final section we wish to discuss briefly the possible extensions of the above analysis and the problems still open.

An important issue we have not touched in this paper is the study of modular invariance. This involves summing the orbifold conformal field theories corresponding to all spin and twist structures and decomposing the resulting state $|\Sigma\rangle$ in a superposition of eigenstates of the operators $b(\kappa)$ and $\bar{b}(\kappa)$. It is important to understand the restrictions imposed by modular invariance on the spectrum of $b(\kappa)$ and $\bar{b}(\kappa)$. This is related to the problem of decomposing $|\Sigma\rangle$ in chiral components, which is relevant for the phenomenologically interesting asymmetric orbifold models [18], and the study of background duality [19].

It would be desirable to work out the analog of the picture changing operation for the bosonic representations. Further, representation changing operators are probably necessary for a rigorous proof of the representation independence. It would also be interesting to formulate a twisted supergeometry, which is needed for the appropriate supersymmetric generalization. We leave these tasks to future work.

One of the relevant traits of our analysis is that the Grassmannian approach essentially applies only to the quantum part of the correlation functions. The basic reason for this is that the proof that a set of harmonic functions on a Riemann surface defines an element of the relevant Grassmannian requires in an essential way the use of the Green identity. When multivalued fields are involved one must use an appropriate dissection of the Riemann surface along the branch cuts of the fields. For instanton solutions, the Green identity involves non-vanishing contributions from the branch cuts proportional to the lattice shifts. This is exactly what makes it impossible to prove that these solutions form an isotropic set. This reflects the inherent difficulty of dealing with bosonic twists in a operator framework [7].

\section{Appendix}

In this appendix we shall expound certain results of twisted geometry which, albeit not directly related to the main subject of this paper, are interesting in their own and may be applied in other fields [10]. We shall further sketch a proof of the twisted Serre and Riemann-Roch theorems.

To begin with, we recall some basic facts. The holomorphic line bundles on the compact Riemann surface $\Sigma_{c}$ endowed with the usual operations of tensor multiplication and inversion form a group, the Picard group $\operatorname{Pic}\left(\Sigma_{c}\right)$. Further, there is a natural isomorphism $\delta^{*}$ between the divisor class group $\operatorname{Div}\left(\Sigma_{c}\right)$ and $\operatorname{Pic}\left(\Sigma_{c}\right)$ such that for any divisor $\mathfrak{D}$ in $\operatorname{Div}\left(\Sigma_{c}\right), c_{1}\left(\delta^{*} \mathfrak{d}\right)=-\operatorname{deg}(\mathfrak{D})$, where $c_{1}(\xi)$ denotes the first Chern class of a bundle $\xi$ and $\operatorname{deg}(d)$ the degree of a divisor $\mathfrak{D}$ [11]

Let $\gamma, \gamma_{1}, \gamma_{2}$ be special twist structures on $\Sigma$. We consider the divisor classes $\mathfrak{m}(\gamma), \mathfrak{s}(\gamma)$ and $\mathrm{t}\left(\gamma_{1}, \gamma_{2}\right)$ defined by

$$
\mathscr{I} \mathfrak{m}(\gamma)=\sum_{l=1}^{h} v_{l}(\gamma) \mathscr{I} p_{l}, \quad \operatorname{deg} m(\gamma)=n(\gamma),
$$




$$
\begin{gathered}
\mathfrak{s}(\gamma)=-\sum_{l=1}^{h}\left[\frac{1}{2}-v_{l}(\gamma)\right] p_{l}, \\
\mathfrak{t}\left(\gamma_{1}, \gamma_{2}\right)=-\sum_{l=1}^{h}\left[v_{l}\left(\gamma_{1}\right)+v_{l}\left(\gamma_{2}\right)+\frac{1}{2}\right] p_{l} .
\end{gathered}
$$

Here $[x]$ denotes the integer part of $x . \mathscr{I}$ is the Jacobi map [11]. Equation (A.1a) holds modulo the Jacobian lattice. It is a straightforward consequence of Abel's and Jacobi's theorems [11] that the conditions (A.1a) determine uniquely $m(\gamma)$ as a divisor class. By means of the isomorphism $\delta^{*}$, we define the holomorphic line bundles

$$
\begin{aligned}
m(\gamma) & =\delta^{*} \mathrm{~m}(\gamma), \\
s(\gamma) & =\delta^{*} \mathfrak{s}(\gamma), \\
t\left(\gamma_{1}, \gamma_{2}\right) & =\delta^{*} \mathrm{t}\left(\gamma_{1}, \gamma_{2}\right) .
\end{aligned}
$$

These satisfy the following identities:

$$
\begin{aligned}
m(1) & =1, \\
m\left(\gamma^{-1}\right) & =m(\gamma)^{-1} \otimes s(\gamma), \\
m\left(\gamma_{1} \cdot \gamma_{2}\right) & =m\left(\gamma_{1}\right) \otimes m\left(\gamma_{2}\right) \otimes t\left(\gamma_{1}, \gamma_{2}\right), \\
s(1) & =1, \\
s\left({ }^{-1}\right) & =s(\gamma), \\
t\left(1, \gamma_{2}\right) & =t\left(\gamma_{1}, 1\right)=1, \\
t\left(\gamma_{,} \gamma^{-1}\right) & =s(\gamma)^{-1}, \\
t\left(\gamma_{1}, \gamma_{2}\right) & =t\left(\gamma_{2}, \gamma_{1}\right), \\
t\left(\gamma_{1}, \gamma_{2}\right) \otimes t\left(\gamma_{1} \cdot \gamma_{2}, \gamma_{3}\right) & =t\left(\gamma_{1}, \gamma_{2} \cdot \gamma_{3}\right) \otimes t\left(\gamma_{2}, \gamma_{3}\right) .
\end{aligned}
$$

A twisted line bundle is a pair $(\xi, \gamma)$ formed by an ordinary line bundle $\xi$ of $\operatorname{Pic}\left(\Sigma_{c}\right)$ an a special twist structure $\gamma$. From (A.3a-i), it follows that the set of all twisted line bundles can be given a group structure in two inequivalent ways,

$$
\begin{gathered}
(\xi, \gamma)_{ \pm}^{-1}=\left(\xi^{-1} \otimes s(\gamma)^{ \pm 1}, \gamma^{-1}\right)_{ \pm} \\
\left(\xi_{1}, \gamma_{1}\right)_{ \pm} \cdot\left(\xi_{2}, \gamma_{2}\right)_{ \pm}=\left(\xi_{1} \otimes \xi_{2} \otimes t\left(\gamma_{1}, \gamma_{2}\right)^{ \pm 1}, \gamma_{1} \cdot \gamma_{2}\right)_{ \pm} \cdot
\end{gathered}
$$

Here the subscript \pm only serves the purpose to indicate which type of group operations are applied. Therefore, the twisted line bundles form two distinct groups $\operatorname{Pic}_{ \pm}(\Sigma, \Gamma)$. An ordinary line bundle $\xi$ can be identified with the pair $(\xi, 1)_{ \pm}$. In this way, $\operatorname{Pic}\left(\Sigma_{c}\right)$ becomes a subgroup of both groups $\operatorname{Pic}_{ \pm}(\Sigma, \Gamma)$, and in fact the group operations of $\mathrm{Pic}_{ \pm}(\Sigma, \Gamma)$ reduce into the ordinary bundle operations of $\operatorname{Pic}\left(\Sigma_{c}\right)$ when restricted to $\operatorname{Pic}\left(\Sigma_{c}\right)$. by

There is a natural group homomorphism $\mu_{ \pm}$of $\operatorname{Pic}_{ \pm}(\Sigma, \Gamma)$ into $\operatorname{Pic}\left(\Sigma_{c}\right)$ defined

$$
\mu_{ \pm}(\xi, \gamma)=\xi \otimes m(\gamma)^{\mp 1}
$$


The importance of this homomorphism stems from the following fundamental isomorphism

$$
\mathcal{O}\left(\mu_{ \pm}(\xi, \gamma)\right) \equiv \mathcal{O}^{ \pm}(\xi, \gamma)
$$

(cfr. Eqs. (2.2-3)). From here, the twisted Serre and Riemann-Roch theorems (cfr. Eqs. (2.5-6)) follow from the corresponding standard theorems.

Proof. For any odd spin structure $[\alpha, \beta]$ define [20]

where $\rho \in \mathbf{C}^{g}$ and

$$
f(\gamma)(p)=\frac{\theta[\alpha, \beta](\mathscr{I} p-\rho+\eta(\gamma))}{\theta[\alpha, \beta]\left(\mathscr{I}_{p}-\rho\right)} \cdot \frac{\prod_{m=1}^{|n(\gamma)|} \theta[\alpha, \beta]\left(\mathscr{I}_{p}-\mathscr{I} q_{m}\right)^{\mathrm{sgn} n(\gamma)}}{\prod_{l=1}^{h} \theta[\alpha, \beta]\left(\mathscr{I} p-\mathscr{I} p_{l}\right)^{v_{l}(\gamma)}},
$$

$$
\eta(\gamma)=\operatorname{sign} n(\gamma) \sum_{m=1}^{\ln (\gamma) \mid} \mathscr{I} q_{m}-\sum_{l=1}^{h} v_{l}(\gamma) \mathscr{I} p_{l}
$$

By using the Riemann vanishing Theorem [20], it is easy to prove that there is a meromorphic function $\phi(\gamma)$ on $\Sigma_{c}$ such that the divisor of zeros and poles of integer degree of $\phi(\gamma) f(\gamma)$ is exactly $m(\gamma)$ (cfr. Eq. (A.1a)). Now, recall that [11] $\mathcal{O}\left(\mu_{ \pm}(\xi, \gamma)\right)_{p}=\mathcal{O}\left(\xi \otimes m(\gamma)^{\mp 1}\right)_{p}=\left\{h \in \mathscr{M}(\xi)_{p} \mid\right.$ either $h=0$ or $\left.\mathrm{d}(h)_{p} \geqq \mp \mathfrak{m}(\gamma)_{p}\right\}$ where $\mathfrak{D}(h)$ is the divisor of the section $h$. Define for any $h \in \mathcal{O}\left(\mu_{ \pm}(\xi, \gamma)\right)_{p}$,

$$
\omega_{ \pm}(\gamma)(h)=[\phi(\gamma) f(\gamma)(p)]^{\mp 1} h .
$$

Clearly, $\omega_{ \pm}(\gamma) h \in \mathscr{M}^{ \pm}(\xi, \gamma)_{p}$, but since the divisor of zeros and poles of integer degree of $\phi(\gamma) f(\gamma)$ is $\mathrm{m}(\gamma)$, it follows from (A.8) that $\omega_{ \pm}(\gamma)(h) \in \mathcal{O}^{ \pm}(\xi, \gamma)_{p}$. We thus have a map from $\mathcal{O}\left(\mu_{ \pm}(\xi, \gamma)\right)$ to $\mathcal{O}^{ \pm}(\xi, \gamma)$ which is easily seen to be a sheaf isomorphism.

To conclude, we provide explicit expressions for a basis of \pm -twisted holomorphic functions and spinors for a general twist structure. We denote by $\lambda_{j}$ and $\mu_{j}, j=1, \ldots, g$ the twists around the $a$ - and $b$-cycles of $\Sigma_{c}$. We further set

$$
\begin{gathered}
\Phi_{\gamma}^{ \pm}(p)=\frac{\prod_{m=1}^{|n(\gamma)|} \theta[\alpha, \beta]\left(\mathscr{I} p-\mathscr{I} q_{m}\right)}{\prod_{l=1}^{h} \theta[\alpha, \beta]\left(\mathscr{I} p-\mathscr{I} p_{l}\right)^{ \pm v_{l}(\gamma)}} \\
\eta^{ \pm}(\gamma)=\sum_{m=1}^{\ln (\gamma) \mid} \mathscr{I} q_{m}-( \pm) \sum_{l=1}^{h} v_{l}(\gamma) \mathscr{I} p_{l},
\end{gathered}
$$

where $[\alpha, \beta]$ is any odd spin structure.

Assume first that $|n(\gamma)| \geqq g$. Let $q_{m},|n(\gamma)|-g+1<m \leqq|n(\gamma)|$ be the $g-1$ non-trivial zeros of the theta function $\theta[\alpha, \beta](\mathscr{I} p-\mathscr{I} q)$. As is well-known these zeros do not depend on $q$ [20]. Set for $1 \leqq m \leqq|n(\gamma)|-g+1$,

$$
f_{\gamma m}^{ \pm}(p)=\frac{\theta[\alpha+\lambda, \beta+\mu]\left(\mathscr{I} p-\mathscr{I} q_{m}+\eta^{ \pm}(\gamma)\right)}{\theta[\alpha, \beta]\left(\mathscr{I} p-\mathscr{I} q_{m}\right)} \Phi_{\gamma}^{ \pm}(p)
$$

(cfr. Eqs. (A.7b-c)). Then, it is not difficult to see that, provided $\operatorname{sign} n(\gamma)= \pm 1$, the functions $f_{\gamma m}^{ \pm}$from a basis of \pm -twisted holomorphic functions. 
Next, assume that $|n(\gamma)|>0$. Set for $1 \leqq m \leqq|n(\gamma)|$,

$$
s_{\gamma m}^{ \pm}(p)=\frac{\theta[\alpha+\lambda, \beta+\mu]\left(\mathscr{I} p-\mathscr{I} q_{m}+\eta^{ \pm}(\gamma)\right)}{E\left(p, q_{m}\right)} \Phi_{\gamma}^{ \pm}(p),
$$

where $E(p, q)$ is the prime form [20]. Then, it is straightforwardly proven that these $1 / 2$ forms form a basis of \pm -twisted holomorphic spinors provided $\operatorname{sign} n(\gamma)= \pm 1$

Acknowledgements. We wish to express our gratitude to T. Jayaraman for helpful discussions and S. Theisen for providing a part of the relevant literature.

Note added in proof. In the preprint version of this paper the definition of the twisted Krichever map was both incorrect and incomplete. This has been correct in the present printed version. Further, the analysis of the instanton contribution outlined above was not contained in the preprint.

After completing this work, we became aware of ref. [21] where a similar formalism for the treatment of multivalued conformal fields on Riemann surfaces is expounded.

\section{References}

1. Gross, D. J., Harvey, J. A., Martinec, E., Rohm, R.: Nucl. Phys. B256, 1985, 253 and Nucl. Phys. B267, 75 (1985)

2. Sen, A.: Phys. Rev. D32, 2102 (1985) and Phys. Rev. Lett. 55, 1846 (1985)

3. Candelas, P., Horowitz, G., Strominger, A., Witten, E.: Nucl. Phys. B258, 285 (1985)

4. Dixon, L. J., Harvey, J. A., Vafa, C., Witten, E.: Nucl. Phys. B261, 687 (1985) and Nucl. Phys. B274, 285 (1986)

5. Alvarez-Gaumé, L., Gomez, C., Reina, C.: Phys. Lett. 190B, 55 (1987) and Proceedings of the trieste spring school of superstrings. Iengo, R., Sezgin, E. (eds.), Singapore: World Scientific 1988; Witten, E.: Commun. Math. Phys. 113, 529 (1988)

6. Vafa, C.: Phys. Lett 190B, 47 (1987); Alvarez-Gaumé, L., Gomez, C., Moore, G., Vafa, C.: Nucl. Phys. B303, 455 (1988); Alvarez-Gaumé, L., Gomez, C., Nelson, P., Sierra, G., Vafa, C.: Nucl. Phys. B311, 333 (1988)

7. Dixon, L. J., Friedan, D., Martinec, E., Shenker, S. H.: Nucl. Phys. B282, 13 (1987)

8. Hamidi, S., Vafa, C.: Nucl. Phys. B279, 465 (1987)

9. Dijkgraaf, R., Verlinde, E., Verlinde, H.: Commun. Math. Phys. 115, 649 (1988)

10. Zucchini, R.: Phys. Lett. B222, 200 (1989)

11. Gunning, R. C.: Lectures on Riemann surfaces. Princeton, NJ: Princeton University Press 1966

12. Atick, J. J., Dixon, L. J., Griffin, P. A., Nemeschansky, D. D.: Nucl. Phys. B298, 1 (1988)

13. Verlinde, E., Verlinde, H.: Nucl. Phys. B288, 357 (1987) and Phys. Lett. B192, 95 (1987)

14. Segal, G., Wilson, G.: Publ. Math. de l'IHES 61, 1 (1985); Pressley, A., Segal, G.: Loop groups. Oxford: Oxford University Press 1986

15. Krichever, I. M.: Russ. Math. Surveys 32, 185 (1977); Dubrovin, B. A., Krichever, I. M., Novikov, S. P.: Topological and algebraic methods in contemporary mathematical physics. Sov. Sci. Reviews 3, I (1982)

16. Vafa, C.: Phys. Lett. B199, 195 (1987)

17. Friedan, D., Martinec, E., Shenker, S. H.: Phys. Lett. 160B, 55(1985) and Nucl. Phys. B271, 93 (1986)

18. Narain, K. S., Sarmadi, M. H., Vafa, C.: Nucl. Phys. B288, 551 (1987)

19. Lauer, J., Zucchini, R.: preprint MPI-PAE/PTh 69/89 and TUM-TH-109/89, to appear in Phys. Lett. B

20. Fay, J. D.: Theta functions on Riemann surfaces, Lectures Notes in Mathematics, vol. 352. Berlin, Heidelberg, New York: Springer 1973

21. Matone, M.: Proceedings of the C. I. M. E., Summer Course in Global Geometry and Mathematical Physics, Montecatini, July, to appear in Lectures Notes in Mathematics. Berlin, Heidelberg, New York: Springer 1988

Communicated by L. Alvarez-Gaumé

Received June 23, 1989 